\title{
Recent advances in computational-analytical integral transforms for convection-diffusion problems
}

\author{
R. M. Cotta ${ }^{1,2}$ - C. P. Naveira-Cotta ${ }^{1}$ D. C. Knupp ${ }^{3}$ - J. L. Z. Zotin ${ }^{4}$ P. C. Pontes ${ }^{1}$. \\ A. P. Almeida ${ }^{1}$
}

Received: 12 April 2017 / Accepted: 6 October 2017 /Published online: 24 October 2017

(C) Springer-Verlag GmbH Germany 2017

\begin{abstract}
An unifying overview of the Generalized Integral Transform Technique (GITT) as a computational-analytical approach for solving convection-diffusion problems is presented. This work is aimed at bringing together some of the most recent developments on both accuracy and convergence improvements on this well-established hybrid numericalanalytical methodology for partial differential equations. Special emphasis is given to novel algorithm implementations, all directly connected to enhancing the eigenfunction expansion basis, such as a single domain reformulation strategy for handling complex geometries, an integral balance scheme in dealing with multiscale problems, the adoption of convective eigenvalue problems in formulations with significant convection effects, and the direct integral transformation of nonlinear convection-diffusion problems based on nonlinear eigenvalue problems. Then, selected examples are presented that illustrate the improvement achieved in each class of extension, in terms of convergence acceleration and accuracy gain, which are related to conjugated heat transfer in complex or multiscale microchannel-substrate geometries, multidimensional Burgers equation model, and
\end{abstract}

R. M. Cotta

cotta@mecanica.coppe.ufrj.br

1 Mechanical Engineering and Nanoengineering Departments - POLI \& COPPE, Federal University of Rio de Janeiro, UFRJ, Rio de Janeiro, Brazil

2 NIDES - CT, Federal University of Rio de Janeiro, UFRJ, Rio de Janeiro, Brazil

3 Mechanical Engineering Department - Polytechnic Institute, State University of Rio de Janeiro, UERJ, Nova Friburgo, Brazil

4 Centro Federal de Educação Tecnológica Celso Suckow da Fonseca, CEFET/RJ, Itaguaí, Brazil diffusive metal extraction through polymeric hollow fiber membranes. Numerical results are reported for each application and, where appropriate, critically compared against the traditional GITT scheme without convergence enhancement schemes and commercial or dedicated purely numerical approaches.

\section{Introduction}

The quest for accuracy and speed in computer simulations of heat and fluid flow problems is clearly endless, as more massive and complex computations are pursued, independent of the mathematical methodology employed. The progress on numerical methods for partial differential equations promoted along the last 60 years or so, closely followed by the sometimes disruptive but continuous improvements on computer hardware, has not only offered the path for simulating threedimensional complex domains and chaotic time evolutions, but also made it feasible to analyze multiple potentials and multiple space and time scales within the same computation. Nevertheless, in different contexts of numerical methods development, gains in accuracy end up by sacrificing speed, while gains in speed, if not obtained by accuracy relaxation, are not always followed by an also desirable gain in accuracy, due to some sort of improvement saturation inherent to the discrete nature of the nowadays well-established purely numerical methods.

Some 30 years after the computer boom and by the end of the computational engineering maturation period, a few different research efforts started promoting the development of hybrid numerical-analytical methods for partial differential equations, in attempts of combining the ample analytical knowledge base already available, with co-currently developed numerical tools for algebraic and ordinary differential 
equations. The aim was to achieve simultaneous and significant gains in both accuracy and speed, as well as increased robustness, at the price of additional analytical effort, while also alleviating somehow the applicability limitations of classical analytical approaches. Numerical inversion of Laplace transforms, Finite analytic methods, and Generalized integral transforms are examples of hybrid methodologies that turned into alternative hybrid approaches, which found their own applicability and relevance in modern engineering practice and research. Almost in parallel, the advancement of symbolic computation platforms has also been playing a major role in the expansion of the development interest and applicability width of such classes of hybrid solution methodologies, drastically reducing the developer or user derivation effort along the analytical phases of these approaches.

The integral transform method is a classical analytical tool for exact solution of certain classes of linear partial differential equations, which has originated from the Separation of Variables method introduced by Fourier [1] in the realm of heat diffusion problems, and has been extensively employed in thermal sciences and engineering for almost 200 years [2-5]. The overall concept is to employ an eigenfunction expansion for the unknown potential, based on the orthogonality property of the eigenvalue problem that results from Separation of Variables as applied to the homogeneous version of the original transient (or steady) linear diffusion problem. Such a natural eigenvalue problem permits, a priori, the construction of both a transformation and an inversion formulae. Thus, upon integral transformation, the space variables are eliminated and the original PDE gives place to a system of decoupled ordinary differential equations for the transformed potentials. This linear decoupled ODE system is then readily solved in analytical form, and the inversion formula allows for the explicit reconstruction of the potential field, via the inversion formula already available upon definition of the eigenvalue problem. This analytical approach has to some extent lost relevance after the appearance of computers and the development of the more flexible numerical methods, but even though, retained a complementary role in the verification of numerical codes and in the realm of applications for a few classes of transformable linear problems $[4,5]$.

Nevertheless, the integral transform method was progressively extended and generalized along the last three decades or so, leading to the establishment of a hybrid numericalanalytical methodology known as the Generalized Integral Transform Technique (GITT) [6-12]. The generalized approach again involves the consideration of an eigenfunction expansion, based on a more freely chosen eigenvalue problem, that desirably retains part of the information on the operators of the original problem, and leads, upon integral transformation, to a coupled linear or nonlinear infinite transformed ODE system, usually to be numerically solved upon truncation to a sufficiently large finite order. Therefore, the more costly numerical task is undertaken essentially in one single independent variable (time for a transient problem or one of the space variables for a steady-state problem), and the original potential solution is analytically recovered in all the other independent variables. This hybrid numerical-analytical method holds the relative merits on robustness and accuracy of the classical analytical technique, with the inherent gain in computational speed in comparison to the conventional purely numerical methods, while extending the applicability and flexibility of an analytic-type methodology towards that of a purely numerical approach. Various classes of problems that could not previously be handled by the classical analytical approach were then progressively dealt with via the generalized method [6-12]. The list of extensions achieved through the GITT includes solving equations with time variable coefficients, moving boundary problems, nonlinear formulations in general, irregular domains, phase change problems, eigenvalue problems, boundary layer and Navier-Stokes equations, etc., as reviewed in different sources [6-14]. In a natural sequence to the handling of such a priori non-transformable classes of diffusion and convection-diffusion problems, the hybrid method was consolidated into a general purpose algorithm, also known as the UNIT (UNified Integral Transforms) algorithm $[13,14]$, in order to facilitate a more widespread use.

While developing and applying such general purpose algorithm, the need for a number of computational improvements and additional theoretical developments became more evident and led to some recent advances on the GITT methodology [15-26], which are here consolidated. Among such recent advancements, one may point out the single domain reformulation strategy for complex geometries, the integral balance approach for convergence enhancement of problems with multiscale or abruptly varying coefficients, the proposition of convective eigenvalue problems for formulations with significant convective effects, and the direct use of nonlinear eigenvalue problems in the integral transformation process of nonlinear PDEs [15-26]. All the methodological variants here discussed are aimed at the enrichment of the eigenfunction expansion basis, towards increasing the amount of information from the original formulation that is carried into the eigenvalue problem and then recovered at any spatial position by the corresponding eigenfunctions. The inversion formula, which represents the final solution of the originally posed problem, is essentially composed of the eigenfunctions and the transformed potentials, with the eigenfunctions offering the reconstruction of the local spatial dependence and the transformed potentials covering for the overall time variable (or equivalent space variable) behaviour, while influenced by the space domain integrated operators coefficients. Therefore, the more information that is locally (and even instantaneously, in nonlinear eigenvalue problems) accounted for by the eigenfunctions, some reduction is expected on the 
number of modes that shall be required to represent such spatial effects through the transformed potentials, which, as mentioned before, only perceive the space variables information in integrated form. These ideas are here compiled and linked, providing an unified view of this continuously improving computational-analytical approach. Selected examples are then presented to illustrate such recent developments, that further enhance the integral transforms computational performance.

\section{Computational-analytical approach}

The formal GITT solution to a general nonlinear convectiondiffusion problem is first presented, from which the recently proposed extensions, to be consolidated in what follows, shall become more evident. A transient multidimensional convection-diffusion problem of $n$ coupled potentials is thus considered, defined in the single arbitrary region $V$, with boundary surface $S[12]$ :

$$
\begin{aligned}
w_{k}(\mathbf{x}) L_{k, t} T_{k}(\mathbf{x}, t)=L_{k} T_{k}(\mathbf{x}, t)-\mathbf{u}(\mathbf{x}, t, \mathbf{T}) \cdot \nabla T_{k}(\mathbf{x}, t) \\
+g_{k}(\mathbf{x}, t, \mathbf{T}), \quad \mathbf{x} \in V, \quad t>0, \quad k=1,2, \ldots, n
\end{aligned}
$$

where the $t$ variable operator, $L_{k, t}$, for a parabolic (or parabolic-hyperbolic) formulation may be given by,

$L_{k, t} \equiv \frac{\partial}{\partial t}$

while for an elliptic or hyperbolic formulation it is written as

$L_{k, t} \equiv-a_{k}(t) \frac{\partial}{\partial t}\left[b_{k}(t) \frac{\partial}{\partial t}\right]$

and the remaining space coordinates operator, with diffusion and linear dissipation, is given as

$L_{k} \equiv \nabla \cdot\left(K_{k}(\mathbf{x}) \nabla\right)-d_{k}(\mathbf{x})$

The initial or boundary conditions in the $t$ variable are given, respectively, by

$T_{k}(\mathbf{x}, 0)=f_{k}(\mathbf{x}), \quad \mathbf{x} \in V$, for the parabolic formulation

$$
\begin{aligned}
T_{k}(\mathbf{x}, 0) & =f_{k}(\mathbf{x}), \quad \frac{\partial T_{k}(\mathbf{x}, 0)}{\partial t} \\
& =h_{k}(\mathbf{x}), \quad \mathbf{x} \in V, \text { for the hyperbolic formulation }
\end{aligned}
$$

or

$\left[\lambda_{k, l}+(-1)^{l+1} \gamma_{k, l} \frac{\partial}{\partial t}\right] T_{k}(\mathbf{x}, t)=f_{k, l}(\mathbf{x})$, at $t=t_{l}, l$

$=0,1, \mathbf{x} \in V$, for the elliptic formulation where the coefficients $\lambda_{k, l}$ and $\gamma_{k, l}$ allow for obtaining boundary conditions of first, second and third kinds. It should be noted that for the elliptic problem, the variable $t$ stands for one of the space coordinates, chosen not to be eliminated through integral transformation, while the vector $\mathbf{x}$ stands for the remaining one or two space coordinates.

The boundary conditions in the remaining coordinates surfaces are concisely written as

$B_{k} T_{k}(\mathbf{x}, t)=\phi_{k}(\mathbf{x}, t, \mathbf{T}), \mathbf{x} \in S, t>0$

with the boundary conditions operator

$B_{k} \equiv\left[\alpha_{k}(\mathbf{x})+\beta_{k}(\mathbf{x}) K_{k}(\mathbf{x}) \frac{\partial}{\partial \mathbf{n}}\right]$

where the coefficients $\alpha_{k}$ and $\beta_{k}$ again allow for obtaining boundary conditions of the different types, $\mathbf{n}$ denotes the outward-drawn normal to the surface $S$, and the coupled potentials vector is given by

$\mathbf{T}=\left\{T_{1}, T_{2}, \ldots, T_{k}, \ldots, T_{n}\right\}$

Equations (1) offer a more general formulation then it might appear at first glance, since nonlinear terms may be grouped into the equations and boundary conditions source terms, $g_{k}(\mathbf{x}$, $t, \mathbf{T})$ and $\phi_{k}(\mathbf{x}, t, \mathbf{T})$, including even the nonlinear convection terms explicitly shown in Eq. (1a). Thus, the linear coefficients that appear in the different operators above can be interpreted as characteristic ones, chosen so as to provide an informative basis for the eigenfunction expansions to be considered. In the case of decoupled linear source terms, i.e., $g \equiv g(\mathbf{x}, t)$, and $\phi \equiv \phi(\mathbf{x}, t)$, and in the absence of the convective term $(\mathbf{u} \equiv 0)$, this example is reduced to a class I linear diffusion problem for each potential, according to the classification in [5], and formal analytical solutions are readily available via the Classical Integral Transform Technique, once the corresponding SturmLiouville eigenvalue problem has been solved for.

Following the formal solution procedure for nonlinear convection-diffusion problems through integral transforms [6-12], one starts with the consideration of eigenfunction expansions for the unknown potentials. The natural eigenvalue problem choice appears from the direct application of the Separation of Variables method to the linear homogeneous purely diffusive version of the proposed problem, such as in the exact solution of the so called Class I problems [5]. For the formulation in Eqs. (1), the suggested set of decoupled auxiliary problems becomes:

$\left[L_{k}+\mu_{k i}^{2} w_{k}(\mathbf{x})\right] \psi_{k i}(\mathbf{x})=0, \quad \mathbf{x} \in V$

$B_{k} \psi_{k i}(\mathbf{x})=0, \quad \mathbf{x} \in S$

where the eigenvalues, $\mu_{k i}$, and associated eigenfunctions, $\psi_{k i}(\mathbf{x})$, are assumed to be known from analytical expressions, for instance obtained through symbolic computation [27] or 
application of the GITT itself, which reduces the differential eigenvalue problem to algebraic matrix eigenvalue problems, upon integral transformation [7, 12].

Making use of the orthogonality property of the eigenfunctions, one readily defines the integral transform pairs:

$\bar{T}_{k i}(t)=\int_{V} w_{k}(\mathbf{x}) \tilde{\psi}_{k i}(\mathbf{x}) T_{k}(\mathbf{x}, t) \mathrm{d} V, \quad$ transforms

$T_{k}(\mathbf{x}, t)=\sum_{i=1}^{\infty} \tilde{\psi}_{k i}(\mathbf{x}) \bar{T}_{k, i}(t), \quad$ inverses

where the symmetric kernels $\tilde{\psi}_{k i}(\mathbf{x})$ and the associated normalization integrals $N_{k i}$ are given by.

$\tilde{\psi}_{k i}(\mathbf{x})=\frac{\psi_{k i}(\mathbf{x})}{\sqrt{N_{k i}}} ;$ and $N_{k i}=\int_{V} w_{k}(\mathbf{x}) \psi_{k i}^{2}(\mathbf{x}) \mathrm{d} V$

The integral transformation of Eq. (1a) is accomplished by applying the operator $\int_{V} \tilde{\psi}_{k i}(\mathbf{x})(\cdot) \mathrm{d} V$ and making use of the boundary conditions given by Eqs. (1i) and (2b), yielding:

$L_{k, t} \bar{T}_{k i}(t)+\mu_{k i}^{2} \bar{T}_{k i}(t)=\bar{g}_{k i}(t, \overline{\mathbf{T}}), i=1,2, \ldots, \quad t>0, k=1,2, \ldots, n$

where the transformed source term $\bar{g}_{k i}(t, \overline{\mathbf{T}})$ is due to the integral transformation of the equation source terms, and in addition the contribution of the boundary source terms [7, 12], to yield:

$$
\begin{aligned}
\bar{g}_{k i}(t, \overline{\mathbf{T}})= & \int_{V} \tilde{\psi}_{k i}(\mathbf{x})\left[-\mathbf{u}(\mathbf{x}, t, \mathbf{T}) \cdot \nabla T_{k}(\mathbf{x}, t)+g_{k}(\mathbf{x}, t, \mathbf{T})\right] d V \\
& +\int_{S} \phi_{k}(\mathbf{x}, t, \mathbf{T})\left[\frac{\tilde{\psi}_{k i}(\mathbf{x})-K_{k}(\mathbf{x}) \frac{\partial \tilde{\psi}_{k i}(\mathbf{x})}{\partial \mathbf{n}}}{\alpha_{k}(\mathbf{x})+\beta_{k}(\mathbf{x})}\right] d S
\end{aligned}
$$

The initial or boundary conditions in the $t$ variable given by Eqs. (1e-1h) are transformed through the operator $\int_{V} w_{k}(\mathbf{x}) \tilde{\psi}_{k i}$ $(\mathbf{x})(\cdot) d V$, to provide:

$$
\bar{T}_{k i}(0)=\bar{f}_{k i} \equiv \int_{V} w_{k}(\mathbf{x}) \tilde{\psi}_{k i}(\mathbf{x}) f_{k}(\mathbf{x}) d V \text {, for the parabolic problem }
$$

$$
\begin{aligned}
& \begin{aligned}
& \bar{T}_{k i}(0)=\bar{f}_{k i} ;\left.\frac{d \bar{T}_{k i}}{d t}\right|_{t=0} \\
& \quad\left.\bar{h}_{k i} \equiv \int_{V} w_{k}(\mathbf{x}) \tilde{\psi}_{k i}(\mathbf{x}) h_{k}(\mathbf{x}) d V, \text { for }, \mathrm{e}\right) \\
& {\left[\lambda_{k, l}+(-1)^{l+1} \gamma_{k, l} \frac{d}{d t}\right] \bar{T}_{k i}(t)=\bar{f}_{k, l i} } \\
& \equiv \int_{V} w_{k}(\mathbf{x}) \tilde{\psi}_{k i}(\mathbf{x}) f_{k, l}(\mathbf{x}) d V \text {, at } t=t_{l}, l=0,1, \text { for the elliptic problem }
\end{aligned}
\end{aligned}
$$

In obtaining the surface integral term of Eq. (4b), the 2nd Green's formula is employed to express the volume integral involving the transformation of the diffusion term $[4,5]$, in the form:

$$
\begin{aligned}
\int_{V}\left[\tilde{\psi}_{k i}(\mathbf{x}) \nabla \cdot\left(K_{k}(\mathbf{x}) \nabla T_{k}(\mathbf{x}, t)\right)-T_{k}(\mathbf{x}, t) \nabla \cdot\left(K_{k}(\mathbf{x}) \nabla \tilde{\psi}_{k i}(\mathbf{x})\right)\right] \mathrm{d} V= \\
=\int_{S} K_{k}(\mathbf{x})\left[\tilde{\psi}_{k i}(\mathbf{x}) \frac{\partial T_{k}(\mathbf{x}, t)}{\partial \mathbf{n}}-T_{k}(\mathbf{x}, t) \frac{\partial \tilde{\psi}_{k i}(\mathbf{x})}{\partial \mathbf{n}}\right] d S
\end{aligned}
$$

Then, the integrand in the right hand side is evaluated by manipulating the boundary conditions, Eqs. (1i) and (2b), first by multiplying the first one by $\tilde{\psi}_{k i}(\mathbf{x})$ and the second one by $T_{k}(\mathbf{x}, t)$, and subtracting them, to obtain:

$K_{k}(\mathbf{x})\left[\tilde{\psi}_{k i}(\mathbf{x}) \frac{\partial T_{k}(\mathbf{x}, t)}{\partial \mathbf{n}}-T_{k}(\mathbf{x}, t) \frac{\partial \tilde{\psi}_{k i}(\mathbf{x})}{\partial \mathbf{n}}\right]=\phi_{k}(\mathbf{x}, t, \mathbf{T}) \frac{\left(-K_{k}(\mathbf{x}) \frac{\partial \tilde{\psi}_{k i}(\mathbf{x})}{\partial \mathbf{n}}\right)}{\alpha_{k}(\mathbf{x})}$

Alternatively, the integrand can be evaluated by multiplying Eq. (1i) by $\frac{\partial \tilde{\psi}_{k i}(\mathbf{x})}{\partial \mathbf{n}}$ and multiplying Eq. (2b) by $\frac{\partial T_{k}(\mathbf{x}, t)}{\partial \mathbf{n}}$, which after their subtraction leads to:

$K_{k}(\mathbf{x})\left[\tilde{\psi}_{k i}(\mathbf{x}) \frac{\partial T_{k}(\mathbf{x}, t)}{\partial \mathbf{n}}-T_{k}(\mathbf{x}, t) \frac{\partial \tilde{\psi}_{k i}(\mathbf{x})}{\partial \mathbf{n}}\right]=\phi_{k}(\mathbf{x}, t, \mathbf{T}) \frac{\tilde{\psi}_{k i}(\mathbf{x})}{\beta_{k}(\mathbf{x})}$

The expression given by Eq. (4h) should be avoided when second kind boundary conditions are involved $\left(\alpha_{k}(\mathbf{x}) \equiv 0\right.$, $\beta_{k}(\mathbf{x}) \neq 0$ ), while Eq. (4i) should be avoided for first kind boundary conditions $\left(\alpha_{k}(\mathbf{x}) \neq 0, \beta_{k}(\mathbf{x}) \equiv 0\right)$, or the two formulae can be combined through algebra in the general form of Eq. (4b), valid in any situation.

For the solution of the infinite coupled system of nonlinear ordinary differential equations given by Eqs. (4), one usually needs to make use of special numerical algorithms, after the truncation of the system to a sufficiently large finite order. Such ODE systems are likely to present a significant stiffness ratio, especially for larger truncation orders, due to the markedly different $t$ variable behaviour of the lowest and highest order transformed potentials. The built-in routine NDSolve of the Mathematica system [27], for instance, offers automatic control of absolute and relative errors and dedicated schemes for stiff systems, including an automatic stiffness switching procedure. After the transformed potentials have been numerically computed, the Mathematica routine automatically provides an interpolating function object that approximates the $t$ variable behaviour of the solution in a continuous form. Then, the inversion formula can be recalled to analytically and explicitly yield the potential field representation at any desired position $\mathbf{x}$ and time $t$.

An important computational aspect in this hybrid approach is the employment of filtering solutions for convergence 
acceleration $[7,9]$. The aim is to reduce the required truncation orders in the eigenfunction expansions, since this directly affects the size of the truncated transformed ODE system, and its numerical solution represents most of the overall computational effort in this methodology. Therefore, the idea behind filtering is to extract information from the problem formulation, preferably in analytical form, to reduce the magnitude of the original equation and boundary conditions source terms, which are ultimately responsible for the convergence deviation from the exponential decay pattern (spectral convergence). In general form, the filtering is introduced through a readily obtainable function, subtracted from the original potential, as:

$$
T_{k}(\mathbf{x}, t)=T_{k}^{*}(\mathbf{x}, t)+T_{F, k}(\mathbf{x} ; t)
$$

where $T_{F, k}(\mathbf{x} ; t)$ is the proposed filter and $T_{k}{ }^{*}(\mathbf{x}, t)$ is the resulting filtered potential to be determined. After introducing Eq. (5) into Eqs. (1), the filtered problem becomes

$$
\begin{aligned}
w_{k}(\mathbf{x}) L_{k, t} T_{k}^{*}(\mathbf{x}, t)= & L_{k} T_{k}^{*}(\mathbf{x}, t)-\mathbf{u}(\mathbf{x}, t, \mathbf{T}) . \nabla T_{k}^{*}(\mathbf{x}, t) \\
& +g_{k}^{*}(\mathbf{x}, t, \mathbf{T}), \quad \mathbf{x} \in V, \quad t>0, \quad k=1,2, \ldots, n
\end{aligned}
$$

The initial or boundary conditions in the $t$ variable are given, respectively, by

$T_{k}^{*}(\mathbf{x}, 0)=f_{k}^{*}(\mathbf{x}), \quad \mathbf{x} \in V$, for the parabolic formulations

$$
\begin{aligned}
T_{k}^{*}(\mathbf{x}, 0) & =f_{k}^{*}(\mathbf{x}),\left.\quad \frac{\partial T_{k}^{*}(\mathbf{x}, t)}{\partial t}\right|_{t=0} \\
& =h_{k}^{*}(\mathbf{x}), \quad \mathbf{x} \in V, \text { for the hyperbolic formulation }
\end{aligned}
$$

or

$$
\left[\lambda_{k, l}+(-1)^{l+1} \gamma_{k, l} \frac{\partial}{\partial t}\right] T_{k}^{*}(\mathbf{x}, t)=f_{k, l}^{*}(\mathbf{x}), \text { at } t=t_{l}, l
$$

$$
=0,1, \mathbf{x} \in V \text {, for the elliptic formulation }
$$

while the boundary conditions in the remaining coordinates are concisely written as

$$
B_{k} T_{k}^{*}(\mathbf{x}, t)=\phi_{k}^{*}(\mathbf{x}, t, \mathbf{T}), \mathbf{x} \in S, t>0
$$

where the filtered functions become

$$
\begin{aligned}
& f_{k}^{*}(\mathbf{x}) \equiv f_{k}(\mathbf{x})-T_{F, k}(\mathbf{x} ; 0) \\
& h_{k}^{*}(\mathbf{x}) \equiv h_{k}(\mathbf{x})-\left.\frac{\partial T_{F, k}(\mathbf{x} ; t)}{\partial t}\right|_{t=0} \\
& g_{k}^{*}(\mathbf{x}, t, \mathbf{T})= g_{k}(\mathbf{x}, t, \mathbf{T})-w_{k}(\mathbf{x}) L_{k, t} T_{F, k}(\mathbf{x}, t) \\
&+L_{k} T_{F, k}(\mathbf{x}, t)-\mathbf{u}(\mathbf{x}, t, \mathbf{T}) . \nabla T_{F, k}(\mathbf{x}, t)
\end{aligned}
$$

$f_{k, l}^{*}(\mathbf{x})=f_{k, l}(\mathbf{x})^{-}\left[\lambda_{k, l}+(-1)^{l+1} \gamma_{k, l} \frac{\partial}{\partial t}\right] T_{F, k}(\mathbf{x}, t)$, at $t=t_{l}, l=0,1, \mathbf{x} \in V$

$\phi_{k}^{*}(\mathbf{x}, t, \mathbf{T})=\phi_{k}(\mathbf{x}, t, \mathbf{T})-\alpha_{k}(\mathbf{x}) T_{F, k}(\mathbf{x}, t)-\beta_{k}(\mathbf{x}) k_{k}(\mathbf{x}) \frac{\partial T_{F, k}(\mathbf{x} ; t)}{\partial \mathbf{n}}, \quad \mathbf{x} \in S$

In case the filtering solution identically satisfies the boundary conditions, the boundary source term becomes zero, and the filtered equation source term remains as defined in Eq. (7c). Besides the simplest algebraic boundary conditions filters, a number of filtering strategies have been proposed along the GITT development, including recursive filters, localinstantaneous filtering, implicit nonlinear filter, and progressive filtering in multidimensional problems $[9,13,14]$. It is well documented that such strategies are very effective in providing faster converging eigenfunction expansions.

Another important computational aspect of the GITT solution, specifically in multidimensional applications, is the appropriate ordering of the parcels in the infinite summation of the inverse formula, Eq. (3b), in representing the final integral transform solution for the related potential. In multidimensional situations, as a result of the analytical solution of the associated multidimensional eigenvalue problem, the expansion can be expressed as double or triple infinite summations for two or three-dimensional transient problems, respectively. For computational purposes, these nested summations will have to be truncated to finite orders somehow, but if they are truncated to a certain prescribed finite order each, the computations become inefficient and costly. For instance, if the double summation of a two-dimensional eigenfunction expansion is truncated to individual orders $N_{x}$ and $N_{y}$, the terms with indices $\left(1, N_{y}+1\right)$ and $\left(N_{x}+1,1\right)$ will be left out and are certainly more relevant to the final result than the last term accounted for, of indices $\left(N_{x}, N_{y}\right)$, and even several others before that last one. Therefore, for an appropriate computation of these expansions, the infinite multiple summations should first be converted to the single sum representation of Eq. (3b), with the appropriate reordering of terms according to their relative contribution to the final numerical result [9]. Since the final solution is not a priori known, the criteria which shall govern this reordering of the multiple summations into a single one should borrow information from the transformed ODE system formulation. The most common choice of reordering strategy is based on arranging in increasing order the sum of the squared eigenvalues in each spatial coordinate, which result from the transformation of the characteristic diffusive and dissipative operators within $L_{k}$. It offers a good compromise between the overall convergence enhancement and simplicity in use. However, certain applications may require more informative reordering criteria, that accounts for the influence of transformed initial conditions and transformed nonlinear source terms in the ODE system. To more clearly understand the 
possible reordering schemes, one may examine the formal solution of the transformed potentials, Eqs. (4), for the parabolic problem case, which is essentially a nonlinear integral Volterra-type equation, written as:

$$
\begin{aligned}
\bar{T}_{k i}(t)= & \bar{f}_{k i} \exp \left(-\mu_{k i}^{2} t\right) \\
& +\int_{0}^{t} \bar{g}_{k i}\left(t^{\prime}, \overline{\mathbf{T}}\left(t^{\prime}\right)\right) \exp \left[-\mu_{k i}^{2}\left(t-t^{\prime}\right)\right] d t^{\prime}
\end{aligned}
$$

Integration by parts of Eq. (8) provides an alternative expression that allows the understanding of the influence of the transformed initial conditions and source terms in the choice of reordering criteria, in rewriting the multiple series as a single one:

$$
\begin{aligned}
\bar{T}_{k i}(t)=\bar{f}_{k i} \exp \left(-\mu_{k i}^{2} t\right) & +\frac{1}{\mu_{k i}^{2}}\left[\bar{g}_{k i}(t, \overline{\mathbf{T}}(t))-\bar{g}_{k i}(0, \overline{\mathbf{T}}(0)) \exp \left(-\mu_{k i}^{2} t\right)\right] \\
& -\frac{1}{\mu_{k i}^{2}} \int_{0}^{t} \frac{d \bar{g}_{k i}}{d t^{\prime}} \exp \left[-\mu_{i}^{2}\left(t-t^{\prime}\right)\right] d t^{\prime}
\end{aligned}
$$

It is evident that the squared eigenvalues, which involve the combination of the eigenvalues in each spatial coordinate, play a major role in the decay of the absolute values of the transformed potentials, and thus of the infinite summation parcels, both through the exponential term decay exp $\left(-\mu_{k i}^{2} t\right)$ and, for slower convergence rates, through the inverse of the squared eigenvalues, $1 / \mu_{k i}^{2}$. Therefore, the traditionally employed reordering scheme based on the ascending order of the squared eigenvalues should be able to account for some of the most important terms in the adequate reorganization of the expansion. Nevertheless, supposing that the last integral term in Eq. (9a) plays a less important role in the reordering choice, and it even vanishes when the source term is not time dependent, one concludes that the decays of the transformed initial condition and of the transformed source term, play a complementary role in the selection of terms in the eigenfunction expansion for a fixed truncation order. Thus, a more robust selection can be proposed, based on adding to the initially reordered terms, according to the squared eigenvalues criterion, those extra terms that might be of significant contribution to the final result under the analysis of the initial condition decay and of the transformed source term behaviour.

Equation (9a) can be also quite useful in pre-analyzing the convergence behaviour of the expansion at the limits of very small (early transients) or large (steady state) values of the $t$ variable. For instance, the transformed potential solution for the lowest time value of interest, $t=t_{\min }$, may be approximated by the truncated form of Eq. (9a), eliminating the integral term, as:

$$
\begin{aligned}
& \bar{T}_{k i}\left(t_{\min }\right) \cong \bar{f}_{k i} \exp \left(-\mu_{k i}^{2} t_{\min }\right)+\frac{1}{\mu_{k i}^{2}} \\
& \quad \times\left[\bar{g}_{k i}\left(t_{\min }, \overline{\mathbf{f}}\right)-\bar{g}_{k i}(0, \overline{\mathbf{f}}) \exp \left(-\mu_{k i}^{2} t_{\min }\right)\right]
\end{aligned}
$$

where the unknown potentials on the r.h.s. are approximated by the known transformed initial conditions for each potential, $\bar{f}_{k}$. The inverse formula is then recalled, Eq. (3b), and the approximate eigenfunction expansion convergence can be analyzed for each potential in the neighbourhood of the initial condition. This analysis provides not only estimates for the truncation orders to be employed along the transformed ODE system numerical solution, for $t>t_{\min }$, but also aids in understanding the importance of each term in the convergence behaviour. For instance, the criterion that reorders the terms based on the decay of the initial conditions is based on sorting in decreasing order from the expression $\bar{f}_{k i} \exp \left(-\mu_{k i}^{2} t_{\min }\right)$. In the second case, for the general situation of a nonlinear transformed source term, the estimation of the terms importance is more difficult, since the source terms, in the more general nonlinear situation, are not known a priori. As from Eq. (9b), in the vicinity of the initial condition, the transformed source term influence on the convergence rates is clearly analyzed through the approximate term $\frac{\bar{g}_{k i}\left(t_{\min }, \overline{\mathbf{f}}\right)}{\mu_{k i}^{2}}$. However, the source term may have an arbitrary variation along the $t$ variable, especially if it has not been properly filtered, and the reordering around $t=0$ might not be representative of the whole $t$ domain influence. One simple alternative is to consider the limiting case of an uniform unitary source term, representing for instance its normalized maximum value, and analyzing the reordering of terms in descending absolute value based on the expression $\frac{1}{\mu_{k i}^{2}} \int_{V} \tilde{\psi} \sim_{k i}(\mathbf{x}) d V$. Therefore, combining the three criteria, and eliminating the duplicates with respect to the traditional reordering scheme based on the sum of the squared eigenvalues, extra terms might be added to the initially reordered terms that may have relevant effect in the final truncated single summation.

Successive integration by parts of Eq. (8) leads to the general expression below, which finds usefulness in refining the approximate solutions once the $\mathrm{m}^{\text {th }}$ order time derivatives of the transformed source terms, $\bar{g}_{k i}{ }^{(m)}(t) \equiv \frac{d^{m} \bar{g}_{k i}(t)}{d t^{n}}$, can be analytically obtained:

$$
\begin{aligned}
\bar{T}_{k i}(t)= & \bar{f}_{k i} e^{-\mu_{k i}^{2} t} \\
& +\sum_{m=1}^{M} \frac{(-1)^{m+1}}{\mu_{k i}^{2 \cdot m}}\left[\bar{g}_{k i}{ }^{(m-1)}(t)-\bar{g}_{k i}{ }^{(m-1)}(0) e^{-\mu_{k i}^{2} t}\right] \\
& +\frac{(-1)^{M}}{\mu_{k i}^{2 \cdot M}} \int_{0}^{t} e^{-\mu_{k i}^{2}\left(t-t^{\prime}\right)} \bar{g}_{k i}{ }^{(M)}\left(t^{\prime}\right) d t
\end{aligned}
$$

where $M$ is the total number of integration by parts performed. For instance, the second level integration yields the following approximate linearized solution for convergence and 
reordering analysis:

$$
\begin{gathered}
\bar{T}_{k i}\left(t_{\min }\right) \cong \bar{f}_{k i} \exp \left(-\mu_{k i}^{2} t_{\min }\right)+\frac{1}{\mu_{k i}^{2}}\left[\bar{g}_{k i}\left(t_{\min }, \overline{\mathbf{f}}\right)-\bar{g}_{k i}(0, \overline{\mathbf{f}}) \exp \left(-\mu_{k i}^{2} t_{\min }\right)\right] \\
-\frac{1}{\mu_{k i}^{4}}\left[\bar{g}_{k i}^{(1)}\left(t_{\min }, \overline{\mathbf{f}}\right)-\bar{g}_{k i}^{(1)}(0, \overline{\mathbf{f}}) \exp \left(-\mu_{k i}^{2} t_{\min }\right)\right]
\end{gathered}
$$

Symbolic computation tools also allow for the iterative analytical derivation of refined approximate solutions, which may find usefulness even beyond such preliminary convergence and reordering analysis. In addition, such approximate solutions can be employed in the modulation of the transformed potentials, so as to reduce the stiffness ration in the numerical solution of the transformed ODE system, yielding improved computational performance.

\section{GITT algorithm variants}

A few recently introduced variants of the basic computational algorithm are now examined, which particularly enhance the computational performance of the GITT approach in complex geometries and heterogeneous media, multiscale variable properties and/or dimensions, convection-dominated problems, and nonlinear boundary conditions.

The ideas explored below are all related to the proposition of more informative eigenfunction expansion basis, by incorporating into the eigenvalue problem further knowledge on the coefficients of the original equation and boundary conditions operators. The inverse formula, Eq. (3b), is essentially composed of the contributions from the normalized eigenfunctions, which are $\mathbf{x}$ dependent only in this formal solution, and from the transformed potentials, which are $t$ dependent only. The eigenfunctions are orthogonal oscillatory functions with increasing frequency for increasing eigenvalue order (or mode), while the transformed potentials are expected to decay in absolute value with increasing eigenvalue order, as made evident from the formal solution Eq. (8), essentially governing the convergence of the expansion. Any coefficient or operator that is not brought into the eigenvalue problem formulation, after choosing the linear coefficients $w_{k}(\mathbf{x})$, $K_{k}(\mathbf{x}), d_{k}(\mathbf{x}), \alpha_{k}(\mathbf{x}), \beta_{k}(\mathbf{x})$, will become part of the equation and boundary condition source terms. These will then affect the transformed potential, both through their explicit time dependence and through their spatially integrated form in the transformation of the source terms, Eq. (4b), eventually including the inherent nonlinear behaviour. Therefore, a basis with limited information from the original problem formulation, naturally penalizes the convergence behaviour in light of the artificial and relevant source terms that are generated, and also due to the local spatial information on the operators not accounted for in the eigenvalue problem, that will affect the transformed potentials, only in integrated form, in general requiring a large number of modes for recovery of this information. Thus, the more information from the original formulation that is incorporated into the eigenvalue problem, less significant should be the spatial dependence of coefficients and operators of the source terms on the transformed potentials, therefore improving convergence rates. Also, the eigenfunctions should in principle locally perceive the spatial functional information provided by the chosen characteristic coefficients, which are incorporated into the eigenvalue problem. Nevertheless, when the eigenvalue problem itself is handled by GITT, with a simpler auxiliary eigenfunction basis, this spatial dependence again appears only in integrated form in the algebraic eigenvalue problem coefficients, then requiring large truncation orders for convergence of the eigenvalues and eigenfunctions themselves. Anyway, there is generally already a significant gain in bringing more information into the eigenvalue problem, since it is computationally less costly to handle the large order algebraic eigenvalue problem than a large transformed ODE system.

First, a single domain formulation strategy is described, which not only facilitates the handling of complex geometries and heterogeneous media, but also provides an evident path for incorporating the spatial information from various subregions or materials into one single eigenvalue problem with spatially varying coefficients. Second, an integral balance procedure is detailed which leads to the convergence acceleration in the solution of eigenvalue problems with spatially variable coefficients through integral transforms. The aim is to account for the local spatial variation of these coefficients in the form of an improved inverse formula for the eigenfunction, thus improving convergence with respect to the space variability representation solely via the integrated form present in the algebraic eigenvalue problem coefficients. Third, an algebraic transformation is presented that allows for the incorporation of convective operators into a generalized diffusion operator with more complex space dependence, thus permitting the convective effects to be directly influent on the eigenfunctions spatial behaviour and also reducing the importance of convection in the source terms. Fourth, a very promising variant is illustrated, through consideration of any nonlinear operator coefficient in the boundary conditions directly into the eigenvalue problem formulation. This approach allows for an optimum convergence rate at the nonlinear boundaries, which would otherwise markedly penalize convergence in the form of a boundary source term only accounted for by the transformed source that results from the 2nd Green's identity. In this case, the nonlinear eigenfunction will inherently be both space and time dependent, thus sharing with the transformed potentials the response to the nonlinear effects. All such ideas have a direct effect on both accuracy and convergence rates, while opening new perspectives for extension of the hybrid methodology. 


\subsection{Single domain formulation strategy}

Consider the general parabolic formulation from Eqs. (1), defined in a multiregion configuration that is formed by $n_{V}$ different sub-regions of volumes $V_{l}, l=1,2, \ldots, n_{V}$, with potential and flux continuity at the interfaces, as illustrated in Fig. 1a [12]. We consider that a certain number of potentials are to be calculated in each sub-region, $T_{k, l}(\mathbf{x}, t), k=1,2, \ldots$, $n$, governed by the corresponding equation and boundary source terms, respectively, $g_{k, l}(\mathbf{x}, t, \mathbf{T})$ and $\phi_{k, l}(\mathbf{x}, t, \mathbf{T})$. Here, for conciseness, the nonlinear equation source term, $g_{k, l}(\mathbf{x}, t$, $\mathbf{T}$ ), already incorporates the nonlinear convective term of Eq. (1a), in the form:

$$
\begin{gathered}
w_{k, l}(\mathbf{x}) \frac{\partial T_{k, l}(\mathbf{x}, t)}{\partial t}=\nabla \cdot\left(K_{k, l}(\mathbf{x}) \nabla T_{k, l}(\mathbf{x}, t)\right)-d_{k, l}(\mathbf{x}) T_{k, l}(\mathbf{x}, t)+g_{k, l}(\mathbf{x}, t, \mathbf{T}), \\
\quad \mathbf{x} \in V_{l}, \quad t>0, \quad k=1,2, \ldots, n, \quad l=1,2, \ldots, n_{V}
\end{gathered}
$$

with initial, interface and boundary conditions given, respectively, by

$$
\begin{aligned}
& T_{k, l}(\mathbf{x}, 0)=f_{k, l}(\mathbf{x}), \quad \mathbf{x} \in V_{l} \\
& T_{k, l}(\mathbf{x}, t)=T_{k, m}(\mathbf{x}, t), \mathbf{x} \in S_{l, m}, t>0 \\
& K_{k, l}(\mathbf{x}) \frac{\partial T_{k, l}(\mathbf{x}, t)}{\partial \mathbf{n}}=K_{k, m}(\mathbf{x}) \frac{\partial T_{k, m}(\mathbf{x}, t)}{\partial \mathbf{n}}, \mathbf{x} \in S_{l, m}, t>0 \\
& {\left[\alpha_{k, l}(\mathbf{x})+\beta_{k, l}(\mathbf{x}) K_{k, l}(\mathbf{x}) \frac{\partial}{\partial \mathbf{n}}\right] T_{k, l}(\mathbf{x}, t)=\phi_{k, l}(\mathbf{x}, t, \mathbf{T}), \mathbf{x} \in S_{l}, t>0}
\end{aligned}
$$

where $\mathbf{n}$ denotes the outward-drawn normal to the interfaces among the sub-regions, $S_{l, m}$, and to the external surfaces, $S_{l}$.

The traditional formalism on the GITT [6-14] would then involve the proposition of a multiregion eigenfunction expansion basis for the integral transform solution of Eqs. (11), based on the so called Class II problems in the classification of ref. [5], which would involve a cumbersome eigenvalue problem. Alternatively, the integral transformation process could be undertaken in each sub-region individually, and then coupled through the transformed interface conditions, which would lead to a large number of transformed potentials equations and certainly require convergence acceleration techniques, such as the integral balance procedure. The idea in the single domain formulation is to avoid either approach, and proceed to interpret problem (11) as one single convection-diffusion problem written for a heterogeneous media [29]. This alternative has been recently proposed, in the context of conjugated heat transfer problems [15-21], when fluid and solid regions were treated as a heterogeneous single medium, after defining space variable coefficients for the whole domain, that account for the abrupt variations of thermophysical properties and other coefficients, through the solid-fluid transitions. Figure 1 provides two possibilities for representation of the single domain, either by keeping the external borders of the original overall domain, after definition of the space variable coefficients, as shown in Fig. 1b, or, if desired, by considering a regular overall domain contour that envelopes the original one, as shown in Fig. 1c. Irregular domains can be directly integral transformed $[7,11]$ for a general region $V$ and, in principle, there is no need to consider

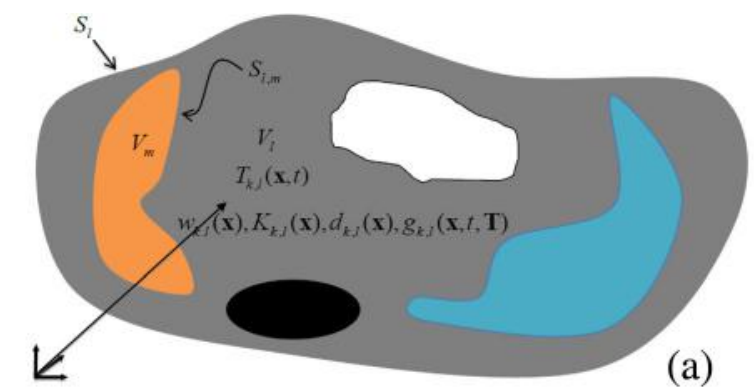

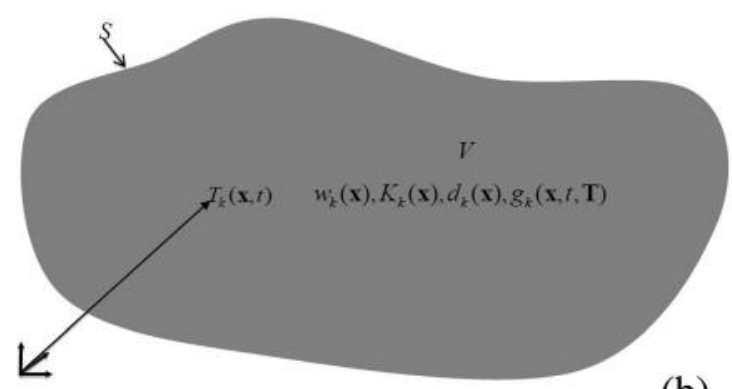

(b)

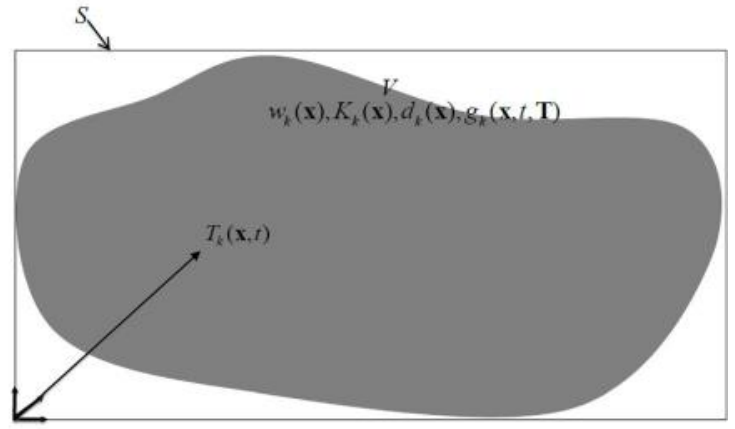

(c)

Fig. 1 a Diffusion or convection-diffusion in a complex multidimensional configuration with $n_{V}$ sub-regions; (b) Single domain representation keeping the original overall domain; (c) Single domain representation considering a regular overall domain enveloping original one 
the second representation possibility pointed out above, except for simplifying the corresponding volume integrals in determining the transformed system coefficients.

Therefore, one may rewrite problem (11) as a single domain formulation with space variable coefficients in the associated operators and source terms, in the form:

$$
\begin{aligned}
w_{k}(\mathbf{x}) \frac{\partial T_{k}(\mathbf{x}, t)}{\partial t} & =\nabla \cdot\left(K_{k}(\mathbf{x}) \nabla T_{k}(\mathbf{x}, t)\right)-d_{k}(\mathbf{x}) T_{k}(\mathbf{x}, t) \\
& +g_{k}(\mathbf{x}, t, \mathbf{T}), \quad \mathbf{x} \in V, \quad t>0
\end{aligned}
$$

with initial and boundary conditions given, respectively, by

$$
\begin{aligned}
& T_{k}(\mathbf{x}, 0)=f_{k}(\mathbf{x}), \quad \mathbf{x} \in V \\
& {\left[\alpha_{k}(\mathbf{x})+\beta_{k}(\mathbf{x}) K_{k}(\mathbf{x}) \frac{\partial}{\partial \mathbf{n}}\right] T_{k}(\mathbf{x}, t)=\phi_{k}(\mathbf{x}, t, \mathbf{T}), \mathbf{x} \in S, t>0}
\end{aligned}
$$

where.

$$
V=\sum_{l=1}^{n_{V}} V_{l}, \quad S=\sum_{l=1}^{n_{V}} S_{l}
$$

The space variable coefficients and source terms in Eqs. (12), after dropping the subscript $l$ for the sub-regions $V_{l}$, are then responsible for the transitions among the different subregions and permit the representation of system (11) as a single domain formulation, to be directly handled by integral transforms as described in Section 2. As desired, this variant incorporates into the proposed eigenvalue problem all the spatial variability inherent to the multiregion or multi-materials physical situation.

\subsection{Integral balance approach for eigenvalue problems}

The GITT approach can be itself employed in the hybrid numerical-analytical solution of this eigenvalue problem, as discussed in [6,12], after choosing an auxiliary eigenvalue problem with simpler structure, defined by the coefficients $\hat{w}_{k}$ $(\mathbf{x}), \hat{k}_{k}(\mathbf{x})$, and $\hat{d}_{k}(\mathbf{x})$, which should allow for an analytical solution. The solution of problem (2) is then itself proposed as an eigenfunction expansion:

$$
\begin{aligned}
& \bar{\psi}_{k i, n}=\int_{V} \hat{w}_{k}(\mathbf{x}) \psi_{k i}(\mathbf{x}) \tilde{\Omega} \sim_{k n}(\mathbf{x}) d V, \quad \text { transform } \\
& \psi_{k i}(\mathbf{x})=\sum_{n=1}^{\infty} \tilde{\Omega}_{k n}(\mathbf{x}) \bar{\psi}_{k i, n}, \quad \text { inverse }
\end{aligned}
$$

where the normalized auxiliary eigenfunction and its norm are given by

$$
\begin{aligned}
& \tilde{\Omega} \sim_{k n}(\mathbf{x})=\frac{\Omega_{k n}(\mathbf{x})}{\sqrt{N_{\Omega k n}}} \text {, with } N_{\Omega_{k n}} \\
& =\int_{V} \hat{w}_{k}(\mathbf{x}) \Omega_{k n}^{2}(\mathbf{x}) d V
\end{aligned}
$$

The simpler auxiliary eigenvalue problem is written as:

$$
\begin{aligned}
& \nabla . \hat{k}_{k}(\mathbf{x}) \nabla \Omega_{k n}(\mathbf{x})+\left(\lambda_{k n}^{2} \hat{w}_{k}(\mathbf{x})-\hat{d}_{k}(\mathbf{x})\right) \Omega_{k n}(\mathbf{x}) \\
& \quad=0, \quad \mathbf{x} \in V
\end{aligned}
$$

with boundary conditions

$\alpha_{k}(\mathbf{x}) \Omega_{k n}(\mathbf{x})+\beta_{k}(\mathbf{x}) \hat{k}_{k}(\mathbf{x}) \frac{\partial \Omega_{k n}(\mathbf{x})}{\partial \mathbf{n}}=0, \quad \mathbf{x} \in S$

Equation (2a) is then operated on with $\int_{V} \tilde{\Omega}_{k i}(\mathbf{x})(\cdot) d V$, to yield the transformed algebraic systems:

$\left(\mathbf{A}_{k}+\mathbf{C}_{k}\right)\left\{\overline{\boldsymbol{\Psi}}_{k}\right\}=\mu_{k}^{2} \mathbf{B}_{k}\left\{\overline{\boldsymbol{\Psi}}_{k}\right\}$

with the elements of the M x M matrices given by [12]:

$$
\begin{aligned}
& A_{k, i j}=\int_{S} \frac{\tilde{\Omega} \sim_{k i}(\mathbf{x})-\hat{k}_{k}(\mathbf{x}) \frac{\partial \tilde{\Omega} \sim_{k i}(\mathbf{x})}{\partial \mathbf{n}}}{\alpha_{k}(\mathbf{x})+\beta_{k}(\mathbf{x})}\left[\beta_{k}(\mathbf{x})\left(k_{k}(\mathbf{x})-\hat{k}_{k}(\mathbf{x})\right) \frac{\partial \tilde{\Omega} \sim_{k j}(\mathbf{x})}{\partial \mathbf{n}}\right] \\
& d S-\int_{S}\left(k_{k}(\mathbf{x})-\hat{k}_{k}(\mathbf{x})\right) \tilde{\Omega} \sim_{k i}(\mathbf{x}) \frac{\partial \tilde{\Omega} \sim_{k j}(\mathbf{x})}{\partial \mathbf{n}} d S+ \\
& +\int_{V}\left(k_{k}(\mathbf{x})-\hat{k}_{k}(\mathbf{x})\right) \nabla \tilde{\Omega} \sim_{k i}(\mathbf{x}) \cdot \nabla \tilde{\Omega} \sim_{k j}(\mathbf{x}) d V+\int_{V}\left(d_{k}(\mathbf{x})-\hat{d}_{k}(\mathbf{x})\right) \tilde{\Omega} \sim_{k i}(\mathbf{x}) \tilde{\Omega} \sim_{k j}(\mathbf{x}) d V \\
& C_{k, i j}=\lambda_{k i}^{2} \delta_{i j} \quad(15 \mathrm{~b}) \\
& B_{k, i j}=\int_{V} w_{k}(\mathbf{x}) \tilde{\Omega}_{k i}(\mathbf{x}) \tilde{\Omega}_{k j}(\mathbf{x}) d V \quad(15 \mathrm{c}, \mathrm{d})
\end{aligned}
$$

where $\delta_{i j}$ is the Kronecker delta.

Therefore, the eigenvalue problem given by Eqs. (2) is reduced to the standard algebraic eigenvalue problems given by Eqs. (15), which can be solved with existing software for matrix eigensystem analysis, yielding the eigenvalues $\mu_{k}$, whereas the corresponding calculated eigenvectors from this numerical solution, $\bar{\psi}_{k i}$, feed the inversion formula, Eq. (13b), to yield the original eigenfunctions.

However, when dealing with the GITT solution of this eigenvalue problem with variable spatial coefficients, it is not always possible to find an auxiliary eigenvalue problem with analytical solution, that incorporates, even partitally, this spatial information, since it may result unsolvable in analytical explicit form. Therefore, in many cases it is required to choose fairly simple expressions for the auxiliary coefficients, $\hat{w}_{k}(\mathbf{x})$ ,$\hat{k}_{k}(\mathbf{x})$, and $\hat{d}_{k}(\mathbf{x})$, which due to their lack of information from the original problem spatial behaviour, may lead to slowly converging expansions for the original eigenfunctions. This is particularly relevant when multiple spatial scales and/or very abrupt variations of the coefficients need to be handled. In such cases, an integral balance procedure [21,22] can be particularly beneficial in accelerating the convergence of such eigenfunction expansions by analytically manipulating the eigenvalue problem formulation, thus rewriting the expansion so as to explicitly account for the local variations of the space variable coefficients. 
The integral balance procedure is a convergence acceleration technique [9] here aimed at obtaining expansions of improved convergence behaviour for both the eigenfunction and its derivatives [21, 22], through integration of the eigenvalue problem over the spatial domain, benefiting from the better convergence characteristics of the integrals of eigenfunction expansions. It consists of the double integration of the original equation that governs the potential for which the convergence improvement is being sought, in this case, the eigenvalue problem itself. Through a single integration of the original equation, in a chosen coordinate, an improved expression for the eigenfunction derivative is obtained, and a second integration then offers an improved relation for computation of the eigenfunction itself. Then, the problem boundary conditions in that coordinate are accounted for, so that the eigenfunctions and respective derivatives at the boundaries can be eliminated. The analytical expressions provided by the integral balance approach can then be employed back into the solution of the eigenvalue problem (2), following the integral transformation procedure above described, yielding an enhanced algebraic eigenvalue problem which provides the eigenvalues and the eigenvectors, to be substituted back in the inversion formula, Eq. (13b). This approach is demonstrated in details in [21, 22], and shall be here illustrated.

\subsection{Convective eigenvalue problem}

An interesting approach towards enhancing convergence of eigenfunction expansions for convection-diffusion problems with relevant convective effects, involves incorporating the convective terms into the chosen eigenvalue problem that forms the basis of the proposed eigenfunction expansion. The aim is to improve convergence by directly accounting for the relative importance of convective and diffusive effects within the eigenfunctions themselves [23, 24], in contrast to the traditional approach when a purely diffusive eigenvalue problem is adopted and the convection terms are incorporated in full within the source terms, as presented above. Through a straightforward transformation of the original convectiondiffusion problem, basically by redefining the coefficients associated with the transient and diffusive terms, the convective terms are merged into a generalized diffusion term with a space variable diffusion coefficient. The generalized diffusion problem then naturally leads to the eigenvalue problem to be adopted for deriving the eigenfunction expansion in the linear situation, as well as for the appropriate linearized version in the case of a nonlinear application. The resulting eigenvalue problem with space variable coefficients is then solved through the GITT, yielding the corresponding algebraic eigenvalue problem upon selection of a simple auxiliary eigenvalue problem of known analytical solution, such as discussed in the previous section. The GITT is also applied in the solution of the generalized diffusion problem, and the resulting transformed ordinary differential equations system is solved either analytically, for the linear case, or numerically for the nonlinear formulation.

This approach is here briefly illustrated by considering Eq. (1a) for a nonlinear parabolic problem and, for conciseness, dropping the subscript $k$ for the potential:

$$
\begin{aligned}
w & (\mathbf{x}) \frac{\partial T(\mathbf{x}, t)}{\partial t}+\mathbf{u}(\mathbf{x}) \cdot \nabla T(\mathbf{x}, t) \\
& =\nabla \cdot[k(\mathbf{x}) \nabla T(\mathbf{x}, t)]-d(\mathbf{x}) T(\mathbf{x}, t)+g(\mathbf{x}, t, T), \quad \mathbf{x} \in V, t>0
\end{aligned}
$$

where $\mathbf{u}(\mathbf{x})$ is a characteristic linear representation of the convective term coefficient, while the remaining of the nonlinear convective operator (or of any other operator) is incorporated into the nonlinear source term, $g(\mathbf{x}, t, \mathbf{T})$, as previously discussed. Equation (16a) can be readily rewritten as a generalized diffusion problem, through a simple transformation of the diffusive and transient terms. The first step in this transformation is to expand the diffusive term in Eq. (16a), to yield:

$$
\begin{aligned}
w(\mathbf{x}) & \frac{\partial T(\mathbf{x}, t)}{\partial t}+\mathbf{u}(\mathbf{x}) \cdot \nabla T(\mathbf{x}, t) \\
= & k(\mathbf{x}) \nabla^{2} T(\mathbf{x}, t)+\nabla k(\mathbf{x}) \cdot \nabla T(\mathbf{x}, t)-d(\mathbf{x}) T(\mathbf{x}, t) \\
& +g(\mathbf{x}, t, T)
\end{aligned}
$$

Next, Eq. (16b) is divided through by $k(\mathbf{x})$ and the convective term is modified to incorporate the second portion of the diffusion operator, as:

$$
\begin{aligned}
& w^{*}(\mathbf{x}) \frac{\partial T(\mathbf{x}, t)}{\partial t}+\mathbf{u}^{*}(\mathbf{x}) \cdot \nabla T(\mathbf{x}, t) \\
& \quad=\nabla^{2} T(\mathbf{x}, t)-d^{*}(\mathbf{x}) T(\mathbf{x}, t)+g^{*}(\mathbf{x}, t, T)
\end{aligned}
$$

where,

$$
\begin{aligned}
w^{*}(\mathbf{x}) & =w(\mathbf{x}) / k(\mathbf{x}) ; d^{*}(\mathbf{x}) \\
& =d(\mathbf{x}) / k(\mathbf{x}) ; g^{*}(\mathbf{x}, t, T) \\
& =g(\mathbf{x}, t, T) / k(\mathbf{x}) ; \mathbf{u}^{*}(\mathbf{x}) \\
& =\frac{1}{k(\mathbf{x})}[\mathbf{u}(\mathbf{x})-\nabla k(\mathbf{x})] ;
\end{aligned}
$$

The modified convective term coefficient vector $\mathbf{u}^{*}$ can be represented in the three-dimensional situation by the three components $\left\{u_{x}^{*}(\mathbf{x}), u_{y}^{*}(\mathbf{x}), u_{z}^{*}(\mathbf{x})\right\}$, in the Cartesian coordinates system with $\mathbf{x}=\{x, y, z\}$, and by defining the exponential transformation coefficients as:

$\hat{k}_{x}(\mathbf{x})=e^{-\int u_{x}^{*}(\mathbf{x}) d x}, \hat{k}_{y}(\mathbf{x})=e^{-\int u_{y}^{*}(\mathbf{x}) d y}, \hat{k}_{z}(\mathbf{x})=e^{-\int u_{z}^{*}(\mathbf{x}) d z}(17 \mathrm{a}-\mathrm{c})$

then the generalized diffusion equation is given as 


$$
\begin{aligned}
\hat{w}(\mathbf{x}) \frac{\partial T(\mathbf{x}, t)}{\partial t}=\hat{k}_{y}(\mathbf{x}) \hat{k}_{z}(\mathbf{x}) \frac{\partial}{\partial x}\left[\hat{k}_{x}(\mathbf{x}) \frac{\partial T(\mathbf{x}, t)}{\partial x}\right] \\
+\hat{k}_{x}(\mathbf{x}) \hat{k}_{z}(\mathbf{x}) \frac{\partial}{\partial y}\left[\hat{k}_{y}(\mathbf{x}) \frac{\partial T(\mathbf{x}, t)}{\partial y}\right]+ \\
\quad+\hat{k}_{x}(\mathbf{x}) \hat{k}_{y}(\mathbf{x}) \frac{\partial}{\partial z}\left[\hat{k}_{z}(\mathbf{x}) \frac{\partial T(\mathbf{x}, t)}{\partial z}\right]-\hat{d}(\mathbf{x}) T(\mathbf{x}, t) \\
+\hat{g}(\mathbf{x}, t, T), \mathbf{x} \in V, t>0
\end{aligned}
$$

where

$$
\begin{aligned}
\hat{k}(\mathbf{x}) & =\hat{k}_{x}(\mathbf{x}) \hat{k}_{y}(\mathbf{x}) \hat{k}_{z}(\mathbf{x}) ; \hat{w}(\mathbf{x})=w^{*}(\mathbf{x}) \hat{k}(\mathbf{x}) ; \hat{\vec{d}}(\mathbf{x}) \\
& =d^{*}(\mathbf{x}) \hat{k}(\mathbf{x}) ; \hat{g}(\mathbf{x}, t, T)=g^{*}(\mathbf{x}, t, T) \hat{k}(\mathbf{x}) \quad(17 \mathrm{e}-\mathrm{h})
\end{aligned}
$$

Separation of Variables as applied to the homogeneous version of Eq. (17d), leads to a non-self-adjoint eigenvalue problem, hence the eigenfunctions are not a priori orthogonal and the classical integral transformation approach is not directly applicable in the form previously presented. However, the Generalized Integral Transform Technique (GITT) [6-14] can still be employed with an appropriate choice of a self-adjoint eigenvalue problem for the eigenfunction expansion basis. When the transformed diffusion coefficients are functions of only the corresponding space coordinate, or $\hat{k}_{x}(\mathbf{x})=\hat{k}_{x}(x), \hat{k}_{y}(\mathbf{x})=\hat{k}_{y}(y), \hat{k}_{z}(\mathbf{x})=$ $\hat{k}_{z}(z)$, with the consequent restrictions on the choices of the characteristic linear coefficients $k(\mathbf{x})$ and $\mathbf{u}(\mathbf{x})$, a generalized diffusion formulation is constructed which leads to a selfadjoint eigenvalue problem, given in such special case by:

$\hat{w}(\mathbf{x}) \frac{\partial T(\mathbf{x}, t)}{\partial t}=\nabla \cdot[\hat{k}(\mathbf{x}) \nabla T(\mathbf{x}, t)]-\hat{a}(\mathbf{x}) T(\mathbf{x}, t)+\hat{g}(\mathbf{x}, t, T), \mathbf{x} \in V, t>0$

where

$\hat{k}(\mathbf{x})=\hat{k}_{x}(x) \hat{k}_{y}(y) \hat{k}_{z}(z)$

and the corresponding self-adjoint eigenvalue problem is written as

$\nabla \cdot[\hat{k}(\mathbf{x}) \nabla \psi(\mathbf{x})]+\left[\mu^{2} \hat{w}(\mathbf{x})-\hat{\hat{a}}(\mathbf{x})\right] \psi(\mathbf{x})=0, \quad \mathbf{x} \in V$

which can be directly solved by the GITT itself, as discussed above. Equation (19) incorporates relevant information on the convective effects, as specified in the chosen linear convective term coefficients that undergo the exponential transformation, which provide a convergence enhancement effect in the integral transform solution.

\subsection{Nonlinear eigenvalue problem}

A whole new frontier for the GITT methodology has been recently envisioned [25], when eigenfunction expansions based on nonlinear eigenvalue problems, that incorporate the original nonlinear equation and boundary condition coefficients, have been proposed. The aim is to achieve improved convergence behaviour, in comparison to the classical approach with a linear eigenvalue problem, here in particular for problems with nonlinear boundary conditions. For the sake of illustration, the nonlinear single potential parabolic version of problem (1) is considered, already with the nonlinear convective term merged into the equation source term, but without merging the nonlinear boundary condition coefficients information into the nonlinear source terms, as previously preferred:

$w(\mathbf{x}) \frac{\partial T(\mathbf{x}, t)}{\partial t}=\nabla \cdot k(\mathbf{x}) \nabla T-d(\mathbf{x}) T+g(\mathbf{x}, t, T), \quad$ in $\mathbf{x} \in V, \quad \mathrm{t}>0$

with initial and boundary conditions

$T(\mathbf{x}, 0)=f(\mathbf{x}), \quad \mathbf{x} \in \mathrm{V}$

$\alpha(\mathbf{x}, t, T) T+\beta(\mathbf{x}, t, T) k(\mathbf{x}) \frac{\partial T}{\partial \mathbf{n}}=\phi(\mathbf{x}, t, T), \quad \mathbf{x} \in \mathrm{S}, \quad \mathrm{t}>0$

where $\alpha$ and $\beta$ are the nonlinear boundary condition coefficients and $\mathbf{n}$ is the outward drawn normal vector to surface $\mathrm{S}$. All the boundary condition coefficients and source terms are allowed to be nonlinear, besides being explicitly dependent also on the space and time variables for the sake of generality.

Here, it suffices to proceed with the formal integral transform solution for the non-filtered potential. Taking a different path from the usual formalism in the GITT, as presented in Section 2, a nonlinear eigenvalue problem that preserves the original boundary condition coefficients is preferred, instead of the one with linear characteristic coefficients, as in Eqs. (2), in the form:

$\nabla . k(\mathbf{x}) \nabla \psi_{i}(\mathbf{x} ; t)+\left[\mu_{i}^{2}(t) w(\mathbf{x})-d(\mathbf{x})\right] \psi_{i}(\mathbf{x} ; t)=0, \mathbf{x} \in \mathrm{V}$

with boundary conditions

$\alpha(\mathbf{x}, t, T) \psi_{i}(\mathbf{x} ; t)+\beta(\mathbf{x}, t, T) \mathrm{k}(\mathbf{x}) \frac{\partial \psi_{i}(\mathbf{x} ; t)}{\partial \mathbf{n}}=0, \mathbf{x} \in \mathrm{S}$

and the solution for the associated $t$-dependent eigenfunctions, $\psi_{i}(\mathbf{x} ; t)$, and eigenvalues, $\mu_{i}(t)$, is at this point assumed to be known.

Problem (21) allows for the definition of the following integral transform pair:

$\bar{T}_{i}(t)=\int_{V} w(\mathbf{x}) \psi_{i}(\mathbf{x} ; t) T(\mathbf{x}, t) d V, \quad$ transform 
$T(\mathbf{x}, t)=\sum_{i=1}^{\infty} \frac{1}{N_{i}(t)} \psi_{i}(\mathbf{x} ; t) \bar{T}_{i}(t)$,

inverse

and the normalization integrals

$N_{i}(t)=\int_{V} w(\mathbf{x}) \psi_{i}^{2}(\mathbf{x} ; t) d V$

After application of the integral transformation procedure, the resulting ODE system for the transformed potentials, $\overline{T_{i}}(t)$, is written as:

$\frac{d \bar{T}_{i}(t)}{d t}+\sum_{j=1}^{\infty} A_{i, j}(t, \overline{\mathbf{T}}) \bar{T}_{j}(t)=\bar{g}_{i}(t, \overline{\mathbf{T}}), \quad t>0, i, j=1,2 \ldots$

with initial conditions

$\bar{T}_{i}(0)=\bar{f}_{i}$

where,

$A_{i, j}(t, \overline{\mathbf{T}})=\delta_{i j} \mu_{i}^{2}(t)+A_{i, j}^{*}(t, \overline{\mathbf{T}})$

and

$$
\begin{aligned}
A_{i, j}^{*}(t, \overline{\mathbf{T}})= & -\frac{1}{N_{j}(t)} \int_{V} w(\mathbf{x}) \frac{\partial}{\partial t}\left[\psi_{i}(\mathbf{x} ; t)\right] \psi_{j}(\mathbf{x} ; t) d V \\
\bar{g}_{i}(t, \overline{\mathbf{T}})= & \int_{V} \psi_{i}(\mathbf{x} ; t) g(\mathbf{x}, t, T) d V \\
& +\int_{S} \phi(\mathbf{x}, t, T)\left(\frac{\psi_{i}(\mathbf{x} ; t)-k(\mathbf{x}) \frac{\partial \psi_{i}}{\partial \mathbf{n}}}{\alpha(\mathbf{x}, t, T)+\beta(\mathbf{x}, t, T)}\right) d S
\end{aligned}
$$

$\bar{f}_{i}=\int_{V} w(\mathbf{x}) \psi_{i}(\mathbf{x} ; 0) f(\mathbf{x}) d V$

The nonlinear coupling coefficients, $A_{i, j}^{*}(t, \overline{\mathbf{T}})$, can be alternatively written in terms of surface integrals by manipulating the eigenvalue problem $(21 \mathrm{a}, \mathrm{b})$ for two arbitrary orders $i$ and $j$, to yield:

$$
\begin{aligned}
& A_{i, j}^{*}(t, \overline{\mathbf{T}})=-\frac{1}{N_{j}(t)} \\
& \quad\left\{\frac{1}{\mu_{i}^{2}-\mu_{j}^{2}} \int_{S} k(\mathbf{x})\left[\frac{\partial \psi_{i}(\mathbf{x} ; t)}{\partial t} \frac{\partial \psi_{j}(\mathbf{x} ; t)}{\partial \mathbf{n}}-\frac{\partial^{2} \psi_{i}(\mathbf{x} ; t)}{\partial \mathbf{n} \partial t} \psi_{j}(\mathbf{x} ; t)\right] d s\right\}
\end{aligned}
$$

and the limit of $\mu_{j} \rightarrow \mu_{i}$ needs to be taken so as to evaluate $A_{i, i}^{*}(t, \overline{\mathbf{T}})$, in the form

$$
\begin{aligned}
& A_{i, i}^{*}(t, \overline{\mathbf{T}})=-\frac{1}{N_{i}(t)} \\
& \quad\left\{\frac{1}{2 \mu_{i}} \int_{S} k(\mathbf{x})\left[\left.\frac{\partial^{2} \psi(\mathbf{x} ; t)}{\partial \mu \partial t}\right|_{\mu_{i}} \frac{\partial \psi_{i}(\mathbf{x} ; t)}{\partial \mathbf{n}}-\left.\frac{\partial^{3} \psi(\mathbf{x} ; t)}{\partial \mu \partial \mathbf{n} \partial t}\right|_{\mu_{i}} \psi_{i}(\mathbf{x} ; t)\right] d s\right\}
\end{aligned}
$$

System (23) can be numerically solved through wellestablished initial value problem solvers [27]. It should be recalled that the eigenvalue problem in Eqs. (21) has now to be solved simultaneously with the transformed system given by Eqs. (23), yielding the time variable eigenfunctions, eigenvalues and norms, as is further discussed in $[25,26]$. The desired final solution is then reconstructed from the inverse formula, Eq. (22b). The GITT itself may be employed in the solution of the nonlinear eigenvalue problem, Eqs. (21). The basic idea is to reduce the eigenvalue problem described by the partial differential equation into a nonlinear algebraic eigenvalue problem, which can be solved in combination with the transformed system (23), forming a differential-algebraic system, also solvable through the Mathematica system [27].

\section{Applications and results}

The developments above discussed are now illustrated through applications dealt with via GITT. The first one is associated with conjugated heat transfer in a non-straight microchannel configuration, which demonstrates both the single domain reformulation strategy as well as the comparison of different reordering schemes on the expansions convergence behaviour. The second application deals with conjugated heat transfer in a microchannel within the slip flow regime, representing a formulation with multiscale spatial variation of thermophysical properties, which benefits from the integral balance approach for convergence enhancement. The third selected problem deals with a two-dimensional Burgers equation classical test case, that allows for the demonstration of the convergence enhancement achievable by the convective eigenvalue problem variant. Finally, the fourth application is related to mass transfer in polymeric membranes for metal extraction, which offers a typical formulation with nonlinear boundary conditions, when the consideration of a nonlinear eigenvalue problem leads to markedly improved computational performance.

\subsection{Conjugated heat transfer in corrugated microchannel}

This example is aimed at illustrating the combination of the single domain formulation with the Generalized Integral Transform Technique in tackling conjugated heat transfer problems in arbitrarily shaped channels and substrates $[16-18,20]$. As an illustration, a two-dimensional corrugated microchannel on a rectangular substrate is considered, as depicted in Fig. 2, undergoing a transient heat transfer process. The channel substrate participates on the heat transfer process through both transversal and longitudinal heat conduction. The single domain dimensionless energy equation can be written as [20]: 


$$
\begin{aligned}
W(X, Y) & \frac{\partial \theta(X, Y, t)}{\partial t}+U(X, Y) W(X, Y) \frac{\partial \theta(X, Y, t)}{\partial X} \\
& +\operatorname{Pe} V(X, Y) W(X, Y) \frac{\partial \theta(X, Y, t)}{\partial Y}= \\
= & \frac{1}{\operatorname{Pe}^{2}} \frac{\partial}{\partial X}\left(K(X, Y) \frac{\partial \theta(X, Y, t)}{\partial X}\right)+\frac{\partial}{\partial Y}\left(K(X, Y) \frac{\partial \theta(X, Y, t)}{\partial Y}\right)
\end{aligned}
$$

with initial and boundary conditions:

$$
\begin{array}{r}
\theta(X, Y, 0)=0 ; \theta(0, Y, t)=0 ; \theta\left(X, L_{y}, t\right) \\
\quad=1 ; \frac{\partial \theta\left(L_{x}, Y, t\right)}{\partial X}=0 ; \frac{\partial \theta(X, 0, t)}{\partial Y}=0
\end{array}
$$

In this formulation, the spatially varying coefficients $U(X$, $Y), V(X, Y), W(X, Y)$ and $K(X, Y)$ are responsible for incorporating the two physical domains (solid and fluid stream) into the single domain model given by Eq. (24a), with abrupt transitions at the interfaces. Besides the assumption of constant physical properties, the inertia terms are disregarded in the flow problem, and the velocity components are computed from the mass balance, starting with a fully developed parabolic profile at the entrance.

Following the formal solution procedure described in Section 2, first a filter is proposed, based on the steady state two-dimensional heat conduction equation, yielding homogeneous boundary conditions in both space coordinates in the filtered problem. In this test case, both eigenvalue problems, with constant and variable coefficients, were considered for comparison purposes.

In order to illustrate some numerical results for conjugated heat transfer in arbitrarily shaped microchannels, the working fluid was taken as water $\left(c_{p, f}=4.11 \times 10^{6} \mathrm{~J} / \mathrm{m}^{3} \mathrm{~K}\right.$ and $k_{f}=$ $0.62 \mathrm{~W} / \mathrm{mK})$ and the substrate as acrylic $\left(c_{p, s}=1.75 \times 10^{6} \mathrm{~J} /\right.$ $\mathrm{m}^{3} \mathrm{~K}$ and $\left.k_{s}=0.19 \mathrm{~W} / \mathrm{mK}\right)$, with $\mathrm{Pe}=0.25$. Tables 1 and 2 present the eigenfunction expansion convergence behavior for the calculated steady state temperature field at some selected points in both the solid and fluid regions, with truncation orders from $N=50$ up to 400 . A convergence to four significant digits for a truncation order of $N<400$ is here demonstrated in the region under analysis. For comparison purposes, the same problem has also been numerically solved via the Finite Element Method, employing the commercial CFD solver COMSOL Multiphysics, and the results are shown in the last rows of Tables 1 and 2, showing an agreement of at least two significant digits in comparison with the converged GITT solution with $N=400$, with relative deviations below $1 \%$. Automatic mesh generation ("physics-controlled mesh") was adopted in the COMSOL simulations, with an element size configuration in the "extremely fine" option.
In order to illustrate the solution behavior throughout the domain, Fig. 3 depicts the calculated steady state temperature field in the corrugated microchannel and substrate. One can clearly notice the effects of the internal convection occurring due to the fluid stream inside the microchannel, distorting the isotherms in the substrate region. The results clearly illustrate that the effects of the conjugated heat transfer are fully captured by the single domain formulation approach.

It is also performed a convergence analysis considering two different reordering schemes to achieve a single sum representation for the inverse formula, namely the traditional scheme through the sum of the squared eigenvalues, and a more elaborate one, considering the most important elements appearing in the diagonal of the coefficients matrix of the transformed problem. In this analysis, a sufficiently large number of diagonal elements of this matrix were calculated and sorted in ascending order, and the first four hundred terms were used for the comparative calculation. It is possible to observe in Fig. 4a,b that a slightly better convergence rate is obtained with the second reordering scheme, which accounts for more information from the non-transformed convective terms in the original formulation.

\subsection{Conjugated heat transfer in microchannels with slip flow}

The application here considered aims at illustrating both the single domain reformulation strategy and the integral balance approach for eigenvalue problems discussed above. The problem considered involves incompressible gas flow in a parallelplates microchannel, supposed to be within the slip flow regime, assuming a fully developed velocity profile at the inlet [21], as schematically presented in Fig. 5.

Consider the following dimensionless groups:

$$
\begin{aligned}
& Z=\frac{z / L_{e}}{\operatorname{RePr}}=\frac{z}{L_{e} \operatorname{Pe}} ; \quad Y=\frac{y}{L_{e}} ; \quad U=\frac{u}{u_{a v}} ; \theta^{*}=\frac{T-T_{w}}{T_{i n}-T_{w}} ; \operatorname{Re}=\frac{u_{a v} L_{e}}{\nu_{f}} ; \operatorname{Pr}=\frac{\nu_{f}}{\alpha_{f}} ; \\
& \mathrm{Pe}=\operatorname{RePr}=\frac{u_{a v} L_{e}}{\alpha_{f}} ; \mathrm{Kn}=\frac{\lambda}{2 L_{f}} ; \quad \beta_{t}=\frac{\left(2-\alpha_{t}\right)}{\alpha_{t}} \frac{2 \gamma}{(\gamma+1)} \frac{1}{\operatorname{Pr}} ; \quad \beta_{v}=\frac{2-\alpha_{m}}{\alpha_{m}}
\end{aligned}
$$

where $\lambda$ is the molecular mean free path, $\gamma$ is the specific heat ratio $\gamma=c_{p} / c_{v}, \alpha_{t}$ is the thermal accommodation coefficient, $\alpha_{m}$ is the tangential momentum accommodation coefficient, $\beta_{t}$ is the wall temperature jump coefficient and $\beta_{v}$ is the wall velocity slip coefficient.

The dimensionless fully developed velocity profile is given by [28]:

$U_{f}(Y)=\frac{6 \mathrm{Kn} \beta_{v}+3\left(1-Y^{2}\right) / 2}{1+6 \mathrm{Kn} \beta_{v}}$

The conjugated heat transfer problem under consideration (conduction - internal convection) can be written as a single 


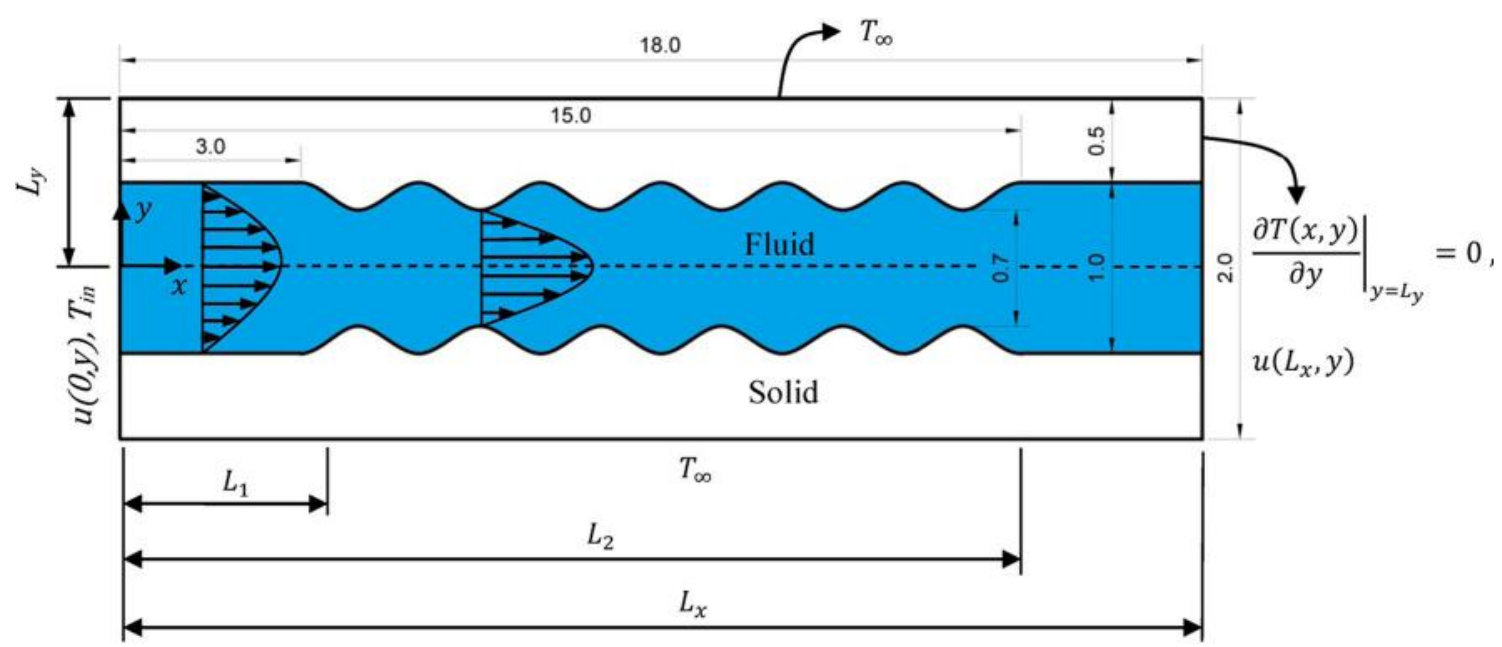

Fig. 2 Conjugated heat transfer for corrugated microchannel in rectangular substrate

domain formulation with space variable coefficients, as discussed in Section 2:

$U(Y) \frac{\partial \theta^{*}(Y, Z)}{\partial Z}=\frac{\partial}{\partial Y}\left(K(Y) \frac{\partial \theta^{*}}{\partial Y}\right), 0<Y<1+L_{f i c}, \quad Z>0$

$\theta^{*}(Y, 0)=1, \quad 0 \leq Y \leq 1+L_{f i c}$

$\left.\frac{\partial \theta^{*}}{\partial Y}\right|_{Y=0}=0, \quad \theta^{*}\left(1+L_{f i c}, Z\right)=0, \quad Z>0$

where:

$U(Y)=\left\{\begin{array}{l}U_{f}(Y), \text { if } 0<Y<Y_{i} \\ 0, \quad \text { if } Y_{i}<Y<1+L_{f i c}\end{array}\right.$

$K(Y)= \begin{cases}1, & \text { if } 0<Y<Y_{i} \\ K_{f i c}, & \text { if } Y_{i}<Y<Y_{i}+L_{f i c} \\ k_{s} / k_{f}, & \text { if } Y_{i}+L_{f i c}<Y<1+L_{f i c}\end{cases}$

From the definition of problem (27), with the space variable coefficients considered, it is clear that a fictitious layer (with thickness $L_{f i c}$ and dimensionless thermal conductivity $K_{f i c}$ ) was artificially inserted in-between the two original domains of the problem (fluid region and channel wall). This

Table 1 Convergence of the GITT solution for steady state temperature in conjugated problem $(X=1, \mathrm{Pe}=0.25)$

\begin{tabular}{llll}
\hline$X=0.1$ & $Y=0.1$ & $Y=0.4$ & $Y=0.7$ \\
\hline$N=50$ & 0.16364 & 0.19294 & 0.40148 \\
$N=120$ & 0.16363 & 0.19294 & 0.40166 \\
$N=190$ & 0.16370 & 0.19295 & 0.40163 \\
$N=260$ & 0.16378 & 0.19303 & 0.40167 \\
$N=330$ & 0.16372 & 0.19309 & 0.40164 \\
$N=400$ & 0.16371 & 0.19308 & 0.40166 \\
COMSOL & 0.16433 & 0.19343 & 0.40248 \\
Relative Deviation & $0.38 \%$ & $0.18 \%$ & $0.20 \%$ \\
\hline
\end{tabular}

fictitious layer is responsible for the simulation of the temperature jump condition in this single domain formulation. The values of $L_{f i c}$ and $K_{f i c}$ are arbitrarily chosen in such a way that the following relation is satisfied:

$\frac{L_{f i c}}{K_{f i c}}=2 \mathrm{Kn} \beta_{t}$

It should be highlighted that axial diffusion effects could be readily dealt with this methodology, but it is here intentionally neglected in such a way that an exact analytical solution is available for this problem [21], as it shall be used as benchmark for the analysis of the results.

The solution of problem (27) can be achieved with the proposition of the following eigenvalue problem, including all the information concerning the transition of the sub-domains, as represented in the space variable coefficients $K(Y)$ and $U(Y)$ :

$\frac{d}{d Y}\left(K(Y) \frac{d \psi_{i}(Y)}{d Y}\right)+\mu_{i}^{2} U(Y) \psi_{i}(Y)=0$

Table 2 Convergence of the GITT solution for steady state temperature in conjugated problem $(X=5, \mathrm{Pe}=0.25)$

\begin{tabular}{llll}
\hline$X=0.25$ & $Y=0.1$ & $Y=0.4$ & $Y=0.7$ \\
\hline$N=50$ & 0.65299 & 0.68514 & 0.82262 \\
$N=120$ & 0.65296 & 0.68469 & 0.82268 \\
$N=190$ & 0.65286 & 0.68479 & 0.82282 \\
$N=260$ & 0.65287 & 0.68486 & 0.82281 \\
$N=330$ & 0.65298 & 0.68496 & 0.82277 \\
$N=400$ & 0.65294 & 0.68503 & 0.82274 \\
COMSOL & 0.65096 & 0.68343 & 0.82339 \\
Relative Deviation & $0.30 \%$ & $0.23 \%$ & $0.08 \%$ \\
\hline
\end{tabular}




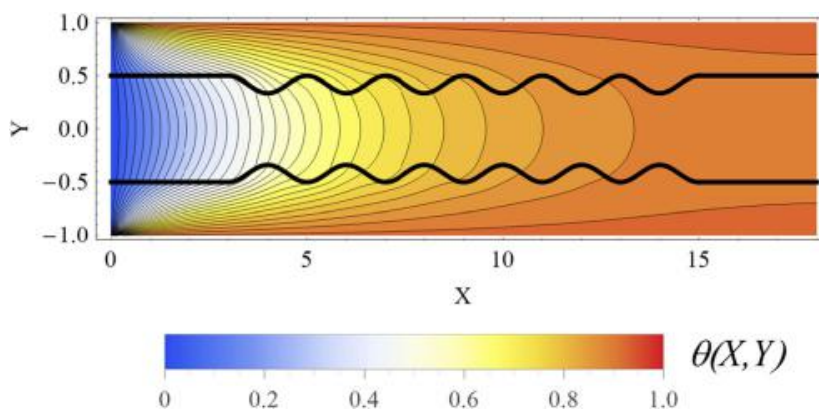

Fig. 3 GITT solution for the steady state temperature in both liquid and solid regions of the microsystem

$\left.\frac{d \psi_{i}}{d Y}\right|_{Y=0}=0 \quad \psi_{i}\left(1+L_{f i c}\right)=0$

Problem (29) does not allow for a closed form solution, but the GITT formalism can be used in order to provide a hybrid numerical-analytical solution, in which Eq. (29a) is integral transformed based on a simpler auxiliary eigenvalue problem. This procedure leads to an algebraic eigenvalue problem, which can be numerically solved after truncation to a sufficiently large finite order [29].

This procedure can be further improved with the integral balance approach for eigenvalue problems [22], previously discussed. Indeed, the main goal of this example is to illustrate this feature as applied to the solution of problem (29), demonstrating the remarkable convergence gains that can be achieved.

Either via the traditional path or via the integral balance approach for eigenvalue problems, once the solution of problem (29) is made available, problem (27) becomes completely transformable, and the analytical solution can be written as:

$\theta^{*}(Y, Z)=\sum_{i=1}^{N} \bar{\theta}_{0, i}^{*} \exp \left(-\mu_{i}^{2} Z\right) \tilde{\psi}_{i}(Y)$

where the inlet condition is transformed as

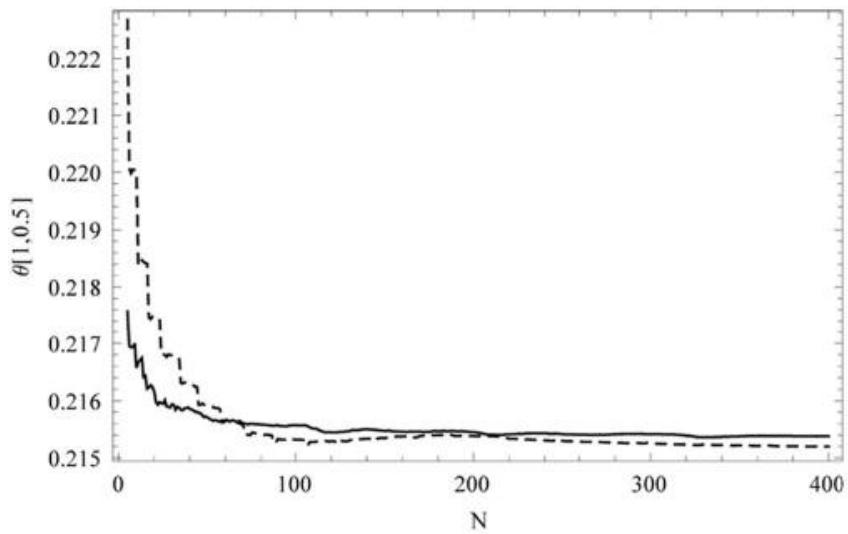

(a) $\bar{\theta}_{0, i}^{*}=\int_{0}^{1+L_{f i c}} U(Y) \tilde{\psi}_{i}(Y) \theta^{*}(Y, 0) d Y$

The final temperature distribution in the original domain can then be readily obtained by simply omitting the fictitious layer from the solution:

$\theta(Y, Z)=\left\{\begin{array}{l}\theta^{*}(Y, Z), \text { if } 0 \leq Y \leq Y_{i} \\ \theta^{*}\left(Y+L_{\text {fic }}, Z\right), \text { if } Y>Y_{i}\end{array}\right.$

The application of the integral balance approach to problem (29), as performed following the general straightforward procedure described in [22], leads to the following improved recursive expressions for the eigenfunctions and their derivatives:

$$
\begin{aligned}
& \psi(Y)=\mu^{2} \int_{Y}^{1+L_{i c}} \frac{1}{K\left(Y^{\prime}\right)}\left[\int_{0}^{Y^{\prime}} U\left(Y^{\prime \prime}\right) \psi\left(Y^{\prime \prime}\right) d Y^{\prime}\right] d Y^{\prime} \\
& \frac{d \psi(Y)}{d Y}=-\frac{\mu^{2}}{K(Y)} \int_{0}^{Y} U\left(Y^{\prime}\right) \psi\left(Y^{\prime}\right) d Y^{\prime}
\end{aligned}
$$

Writing $\psi_{i}(Y)$ as eigenfunction expansions, we have:

$$
\begin{aligned}
& \psi_{i}(Y)=\sum_{n=1}^{\infty} \tilde{\Omega}_{n}(Y) \bar{\psi}_{i, n} \quad \text { inverse } \\
& \bar{\psi}_{i, n}=\int_{0}^{1+L_{f i c}} \psi_{i}(Y) \tilde{\Omega}_{n}(Y) d Y, \quad \text { transform }
\end{aligned}
$$

where.

$\tilde{\Omega}_{n}(Y)=\frac{\Omega_{n}(Y)}{N_{\Omega_{n}}^{1 / 2}}$, with $N_{\Omega_{n}}=\int_{0}^{1+L_{f i c}} \Omega_{n}^{2}(Y) d Y$

with the eigenfunctions $\Omega_{n}(Y)$ obtained from a simpler eigenvalue problem, with closed form solution. The simplest choice, here employed, is:

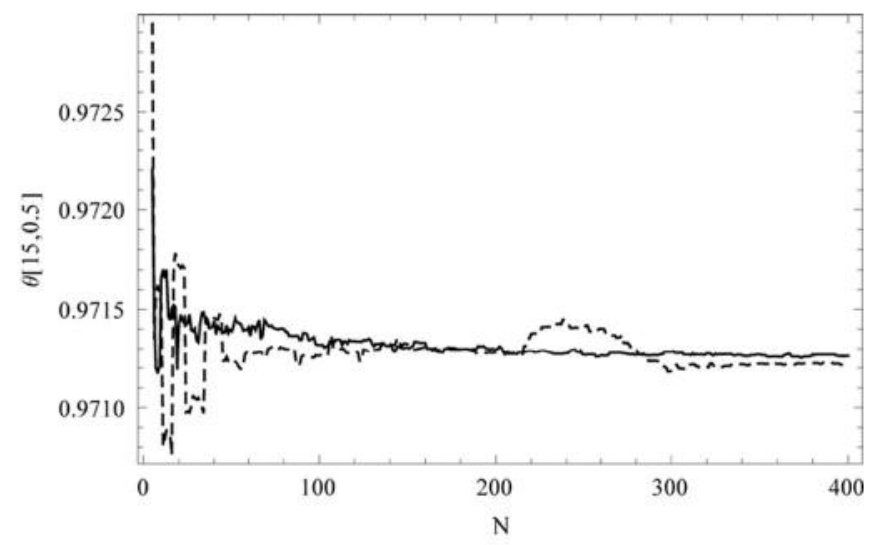

(b)

Fig. 4 Temperature convergence behaviour at $(\mathbf{a})(X, Y)=(1,0.5)$ and $(\mathbf{b})(X, Y)=(15,0.5)$. Dashed line: squared eigenvalues reordering; Solid line: coefficients matrix diagonal reordering 
Fig. 5 Schematic representation of the conjugated heat transfer problem in microchannel with slip flow

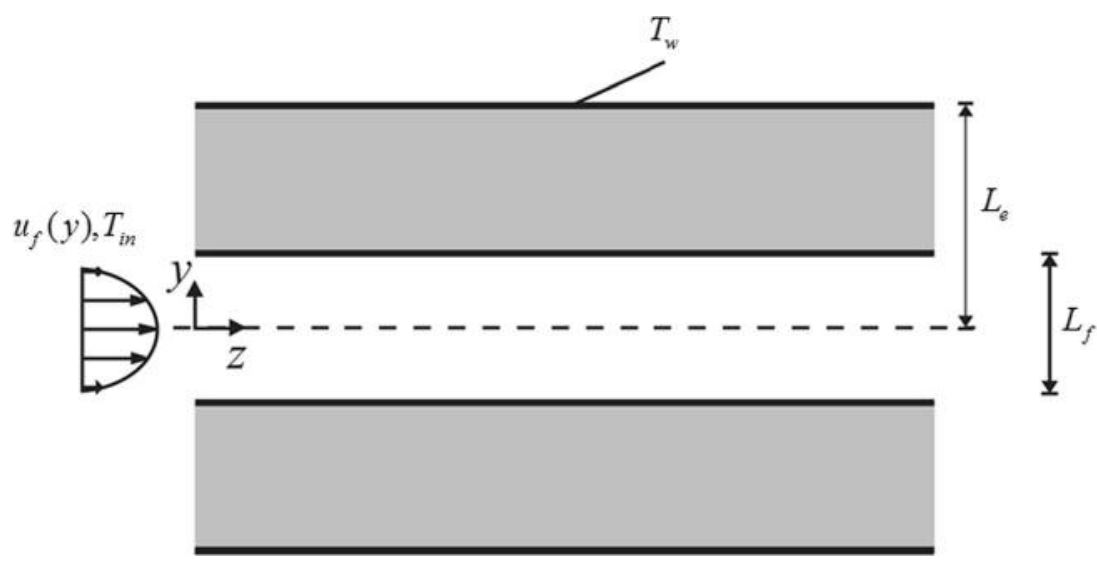

$\left.\frac{d \Omega_{n}}{d Y}\right|_{Y=0}=0, \quad \Omega_{n}\left(1+L_{f i c}\right)=0$

In order to illustrate the remarkable convergence enhancement that is achieved, the results so obtained are compared against those calculated with the traditional GITT solution of the eigenvalue problem. For the numerical results, the dimensionless thermal conductivity has been calculated motivated by an application with a microchannel made of PMMA (polymethyl methacrylate), with $k_{s}=0.2 \mathrm{~W} / \mathrm{m}^{\circ} \mathrm{C}$, with air as the working fluid $\left(k_{f}=0.0271 \mathrm{~W} / \mathrm{m}^{\circ} \mathrm{C}\right)$, so that $k_{s} / k_{f}=7.38$. The fluid region is considered from $Y=0$ to $Y=0.5$, whereas from $Y=0.5$ to $Y=1$ it is considered the channel wall. The remaining slip flow parameters were considered as $\beta_{v}=1.5$, $\beta_{t}=2.0$, and $\mathrm{Kn}=0.025$. For the fictitious layer it has been employed the dimensionless thickness value of $L_{f i c}=0.05$, and the dimensionless thermal conductivity $\left(K_{f i c}\right)$ has been obtained from Eq. (28).

Table 3 presents the convergence behaviour of the temperature distribution considering a fixed truncation order of $N=5$ terms in Eq. (30a) and varying the truncation order of the eigenvalue problem solution (M), here solved employing the traditional GITT solution path [21]. One should observe that in order to obtain accurate results, high truncation orders are needed within the eigenvalue problem solution, such as $\mathrm{M}=300$ terms, in order to achieve an agreement of two significant digits with the exact solution. This slow convergence behaviour takes place due to the multiscale nature of the space variable coefficients present in the eigenvalue problem. It is worth noting that the results are noticeably worse near the interface $(Y=0.5)$, where the abrupt change in the space variable coefficients takes place.

Table 4 presents the convergence behaviour of the temperature distribution at the same positions, but now employing the integral balance approach for the solution of the eigenvalue problem. In this convergence analysis, the truncation order of the final expansion (N), in Eq. (30a), is varied, as well as the truncation order of the eigenvalue problem solution (M). As it can be clearly observed, a remarkable convergence enhancement is achieved, leading to a full convergence of the five significant digits presented with a truncation order as low as $\mathrm{M}=4$, in full agreement with the exact solution employed as 
benchmark. One should also observe that, differently from the traditional approach, the accuracy of the calculated solution is not affected near the interface region $(Y=0.5)$.

\subsection{Two-dimensional Burgers equations}

The use of a convective eigenvalue problem in enhancing convergence is here illustrated through a two-dimensional Burgers equation, allowing for direct comparison against previously published results through the GITT with a purely diffusive eigenfunction basis [13]. The problem here considered is written as [24]:

$$
\begin{aligned}
& \frac{\partial T(x, y, t)}{\partial t}+U_{x}(T) \frac{\partial T(x, y, t)}{\partial x} \\
& +U_{y}(T) \frac{\partial T(x, y, t)}{\partial y}=\frac{\partial^{2} T(x, y, t)}{\partial x^{2}} \\
& +\frac{\partial^{2} T(x, y, t)}{\partial y^{2}}, 0<x<1,0<y<1, t>0
\end{aligned}
$$

with initial and boundary conditions, and the non-linear functions $U_{x}(T)$ and $U_{y}(T)$, given by:

$$
\begin{aligned}
& T(x, y, 0)=1,0 \leq x \leq 1,0 \leq y \leq 1 \\
& T(0, y, t)=0 ; T(1, y, t)=0, t>0 ; \quad T(x, 0, t) \\
& =0 ; T(x, 1, t)=0, t>0 \\
& U_{x}(T)=u_{0}+b_{x} T ; U_{y}(T)=v_{0}+b_{y} T
\end{aligned}
$$

From direct comparison against Eqs. (16-18), taking the linear portion of the velocity coefficients to represent the characteristic convective terms and transporting the remaining terms to the nonlinear source term, the following coefficients correspondence can be reached:

$$
\begin{aligned}
& u_{x}(\mathbf{x})=u_{0} ; v_{x}(\mathbf{x})=v_{0} ; w(\mathbf{x})=1 ; k(\mathbf{x})=1 ; \\
& d(\mathbf{x})=0 ; g(\mathbf{x}, t, T)=-\left(b_{x} T \frac{\partial T}{\partial x}+b_{y} T \frac{\partial T}{\partial y}\right) \\
& \hat{k}_{x}(\mathbf{x})=e^{-u_{0} x} ; \hat{k}_{y}(\mathbf{x})=e^{-v_{0} y} ; \hat{k}(\mathbf{x})=\hat{k}_{x}(x) \hat{k}_{y}(y)=e^{-\left(u_{0} x+v_{0} y\right)} \\
& \hat{w}(\mathbf{x})=\hat{k}(\mathbf{x}) ; \hat{\vec{d}}(\mathbf{x})=0 ; \hat{g}(\mathbf{x}, t, T)=g(\mathbf{x}, t, T) \hat{k}(\mathbf{x}) ;
\end{aligned}
$$

The resulting transformed equation with the generalized diffusion terms representation is then given by:

$$
\begin{aligned}
& \hat{k}(x, y) \frac{\partial T(x, y, t)}{\partial t}= \frac{\partial}{\partial x}\left[\hat{k}(x, y) \frac{\partial T}{\partial x}\right]+\frac{\partial}{\partial y}\left[\hat{k}(x, y) \frac{\partial T}{\partial y}\right] \\
&+\hat{g}(x, y, t, T), 0 \\
&<x<1,0<y<1, t>0
\end{aligned}
$$

which leads to the following eigenvalue problem

$$
\begin{aligned}
& \frac{\partial}{\partial x}\left[\hat{k}(x, y) \frac{\partial \psi}{\partial x}\right]+\frac{\partial}{\partial y}\left[\hat{k}(x, y) \frac{\partial \psi}{\partial y}\right]+\mu^{2} \hat{k}(x, y) \psi(x, y) \\
& \quad=0,0<x<1,0<y<1
\end{aligned}
$$

with boundary conditions

$\psi(0, y)=0 ; \psi(1, y)=0 ; \psi(x, 0)=0 ; \psi(x, 1)=0, t>0$

This two-dimensional eigenvalue problem with space variable coefficients is also readily solved by applying the GITT itself, based on a simpler auxiliary eigenvalue problem. Also, for the special case of a linear formulation, the problem is readily solved in exact form, since the nonlinear source term vanishes, yielding a decoupled transformed ODE system. The traditional solution via a purely diffusive eigenvalue problem [13] can be easily recovered, by adopting $u_{x}(\mathbf{x})=0$ and $v_{x}(\mathbf{x})=$ 0 , and carrying the full convective terms to the source term, i.e., $g(x, y, t)=-\left[U_{x}(T) \frac{\partial T(x, y, t)}{\partial x}+U_{y}(T) \frac{\partial T(x, y, t)}{\partial x}\right]$. Table 5 provides a convergence analysis for $T(x, y, t)$ in the twodimensional Burgers equation, with $b_{x}=0, b_{y}=0$ (linear problem). The three sets of double columns correspond to the values of the velocity coefficients $u_{0}=1, v_{0}=1, u_{0}=10$, $v_{0}=1$, and $u_{0}=10, v_{0}=10$.

For this linear two-dimensional problem, a maximum truncation order of $N=160$ terms has been considered in the solution of the generalized diffusive eigenvalue problem and $N<160$ in computing the potential expansion. The solution of the eigenvalue problems, either for the convective or diffusive basis, employs a reordering scheme based on the sum of the squared eigenvalues of the auxiliary problem. The results in Table 5 for the independent variables $(x=0.1, y=0.1$, $t=0.01$ ), confirm the excellent convergence behavior of the expansions that follow the convective basis proposal, with four converged significant digits at truncation orders as low as $N=60$ when employing the convective basis. The results achieved by the diffusive basis are not even fully converged for the largest truncation order here adopted, $N=160$, and it is noticeable that this solution presents an oscillatory behaviour to reach convergence at the fourth significant digit. It can be observed that since the velocity coefficients are accounted for in the convective eigenvalue problems alternative, the convergence behaviour is not noticeably affected by the increase in their values, while the effect on the convergence rates of the results with a diffusive basis can be clearly observed.

\subsection{Metal separation by polymeric diffusive membranes}

The nonlinear problem that will be here illustrated was proposed in [30] and recently solved by GITT with a simplified nonlinear eigenvalue problem [26]. Here, a more complete 
Table 3 Convergence of the temperature distribution (for $N=5$ ) with respect to the eigenvalue problem truncation order (M) in conjugated problem with slip flow (without convergence enhancement)

\begin{tabular}{|c|c|c|c|c|c|c|}
\hline$M$ & $\theta(0,0.05)$ & Relative deviation & $\theta(0.25,0.05)$ & Relative deviation & $\theta(0.5,0.05)$ & Relative deviation \\
\hline 50 & 0.89526 & $0.36 \%$ & 0.72414 & $1.02 \%$ & 0.29571 & $5.86 \%$ \\
\hline 75 & 0.89589 & $0.29 \%$ & 0.72592 & $0.78 \%$ & 0.30023 & $4.42 \%$ \\
\hline 100 & 0.89674 & $0.20 \%$ & 0.72758 & $0.55 \%$ & 0.30403 & $3.21 \%$ \\
\hline 125 & 0.89705 & $0.16 \%$ & 0.72843 & $0.43 \%$ & 0.30641 & $2.46 \%$ \\
\hline 150 & 0.89730 & $0.13 \%$ & 0.72886 & $0.37 \%$ & 0.30724 & $2.19 \%$ \\
\hline 175 & 0.89750 & $0.11 \%$ & 0.72938 & $0.30 \%$ & 0.30868 & $1.73 \%$ \\
\hline 200 & 0.89760 & $0.10 \%$ & 0.72957 & $0.28 \%$ & 0.30906 & $1.61 \%$ \\
\hline 225 & 0.89772 & $0.09 \%$ & 0.72985 & $0.24 \%$ & 0.30979 & $1.38 \%$ \\
\hline 250 & 0.89779 & $0.08 \%$ & 0.73001 & $0.22 \%$ & 0.31021 & $1.25 \%$ \\
\hline 275 & 0.89785 & $0.07 \%$ & 0.73015 & $0.20 \%$ & 0.31049 & $1.16 \%$ \\
\hline 300 & 0.89791 & $0.06 \%$ & 0.73030 & $0.18 \%$ & 0.31092 & $1.02 \%$ \\
\hline Exact & 0.89849 & - & 0.73159 & - & 0.31412 & - \\
\hline
\end{tabular}

nonlinear eigenfunction expansion basis is adopted, based on Laguerre polynomials that account for the circular tube geometry. It consists of a separation process in a module of supported liquid membranes. The analysis of the performance of the separator is accomplished based on the study of only one membrane, assuming that the others present similar behaviour. A schematic diagram of the hollow fiber membrane is shown in Fig. 6.

The mathematical model is obtained from the mass conservation equations assuming fully developed onedimensional laminar flow of a Newtonian fluid containing the solute to be separated. The fluid enters the separator with known concentration $C_{e}^{*}$ and the separation process starts at $\mathrm{z}=0$, where the fluid comes into contact with the supported liquid membrane. The solute permeates through the liquid membrane by diffusion and on the outside of the fiber reacts instantaneously with the stripping solution, so that its concentration is equal to zero. The distribution coefficient $\mathrm{H}$ is a very important parameter of this mass transfer process, and it is defined as equilibrium distribution ratio of the solute concentration in the liquid membrane to the concentration in the fluid side. In this example, the distribution coefficient will be considered as a linear function of the concentration potential of solute in the aqueous phase. The axial diffusion effect is neglected compared to axial convection and radial diffusion. The dimensionless mathematical model proposed in [26] is given by:

$U(r) \frac{\partial C(r, z)}{\partial z}=\frac{1}{r} \frac{\partial}{\partial r}\left(r \frac{\partial C(r, z)}{\partial r}\right), 0<r<1, z>0$

$C(r, 0)=1,0<r<1$

$\left.\frac{\partial C(r, z)}{\partial r}\right|_{r=0}=0, z>0$

$-\left.\frac{\partial C(r, z)}{\partial r}\right|_{r=1}=S h_{w}(1+\gamma C(1, z)) C(1, z), z>0$

where

$$
\begin{gathered}
r=\frac{r^{*}}{R} ; \quad C(r, z)=\frac{C^{*}}{C_{e}^{*}} ; z=\frac{z^{*} D}{v R^{2}} ; \gamma=\frac{C_{e}^{*} h^{*}}{h_{o}} ; S h_{w}=\frac{k_{w} S R h_{o}}{D} ; \\
U(r)=2\left(1-r^{2}\right) ; s=\frac{L / R_{0}}{\ln \left[1 /\left(1-L / R_{0}\right)\right]} ;
\end{gathered}
$$

Table 4 Convergence of the temperature distribution with respect to the eigenvalue problem and temperature expansion truncation orders (N and $\mathrm{M}$ ) in conjugated problem with slip flow (with integral balance approach)

\begin{tabular}{lllllll}
\hline$N=M$ & $\theta(0,0.05)$ & Relative deviation & $\theta(0.25,0.05)$ & Relative deviation & $\theta(0.5,0.05)$ & Relative deviation \\
\hline 1 & 0.89705 & $0.16 \%$ & 0.72905 & $0.35 \%$ & 0.31532 & $0.38 \%$ \\
2 & 0.90228 & $0.42 \%$ & 0.72862 & $0.41 \%$ & 0.31280 & $0.42 \%$ \\
3 & 0.89870 & $0.02 \%$ & 0.73147 & $0.02 \%$ & 0.31414 & $0.00 \%$ \\
4 & 0.89849 & $0.00 \%$ & 0.73160 & $0.00 \%$ & 0.31412 & $0.00 \%$ \\
5 & 0.89849 & $0.00 \%$ & 0.73160 & $0.00 \%$ & 0.31412 & $0.00 \%$ \\
Exact & 0.89849 & - & 0.73159 & - & 0.31412 & - \\
\hline
\end{tabular}


Table 5 Convergence analysis of eigenfunction expansions with convective and diffusive eigenvalue problems in the solution of the two-dimensional Burgers equation

\begin{tabular}{|c|c|c|c|c|c|c|}
\hline \multirow[t]{3}{*}{$\mathrm{N}$} & \multicolumn{6}{|l|}{$b_{x}=0, b_{y}=0$} \\
\hline & \multicolumn{2}{|l|}{$u_{0}=1, v_{0}=1$} & \multicolumn{2}{|l|}{$u_{0}=10, v_{0}=1$} & \multicolumn{2}{|l|}{$u_{0}=10, v_{0}=10$} \\
\hline & $\mathrm{T}(0.1,0.1,0.01)$ Conv. & $\mathrm{T}(0.1,0.1,0.01)$ Diff. & $\mathrm{T}(0.1,0.1,0.01)$ Conv. & $\mathrm{T}(0.1,0.1,0.01)$ Diff. & $\mathrm{T}(0.1,0.1,0.01)$ Conv. & $\mathrm{T}(0.1,0.1,0.01)$ Diff. \\
\hline 10 & 0.2079 & 0.2118 & 0.1114 & 0.1334 & 0.05472 & 0.06619 \\
\hline 20 & 0.2391 & 0.2440 & 0.1357 & 0.1550 & 0.07683 & 0.10040 \\
\hline 30 & 0.2450 & 0.2451 & 0.1411 & 0.1379 & 0.08114 & 0.07001 \\
\hline 40 & 0.2462 & 0.2469 & 0.1420 & 0.1450 & 0.08188 & 0.08732 \\
\hline 50 & 0.2463 & 0.2457 & 0.1422 & 0.1393 & 0.08208 & 0.07715 \\
\hline 60 & 0.2464 & 0.2458 & 0.1422 & 0.1406 & 0.08211 & 0.08035 \\
\hline 70 & 0.2464 & 0.2458 & 0.1423 & 0.1408 & 0.08212 & 0.08079 \\
\hline 80 & & 0.2458 & 0.1423 & 0.1408 & 0.08212 & 0.08070 \\
\hline 90 & & 0.2458 & & 0.1403 & & 0.07983 \\
\hline 100 & & 0.2459 & & 0.1412 & & 0.08090 \\
\hline 110 & & 0.2460 & & 0.1417 & & 0.08215 \\
\hline 120 & & 0.2460 & & 0.1409 & & 0.08100 \\
\hline 130 & & 0.2460 & & 0.1405 & & 0.07964 \\
\hline 140 & & 0.2461 & & 0.1417 & & 0.08132 \\
\hline 160 & & 0.2463 & & 0.1423 & & 0.08307 \\
\hline
\end{tabular}

Equation (43c) represents the symmetry condition at the channel centerline, while Eq. (43d) imposes the continuity of solute flux across the membrane-fluid interface. Equation (43d) is a nonlinear boundary condition and, therefore, makes it unlikely to obtain a fully analytical solution for this problem.

This same problem has been previously solved by the GITT [31], in its traditional form, by choosing a linear eigenvalue problem as a basis for the eigenfunction expansion, thus without the inclusion of the nonlinear boundary condition in its formulation. Then, from application of Green's second identity, the contribution of the nonlinear boundary source term reappears in the transformed ordinary differential system. However, there is some computational advantage in adopting a nonlinear eigenvalue problem formulation, incorporating the nonlinear boundary condition information directly into the eigenfunction. Following this alternative, the nonlinear eigenvalue problem has been chosen as:

$\frac{\partial}{\partial r}\left[r \frac{\partial \psi_{i}}{\partial r}\right]+\mu_{i}^{2}(z) r U(r) \psi_{i}(r ; z)=0$

$\left.\frac{\partial \psi_{i}}{\partial r}\right|_{r=0}=0 ;\left.\quad \frac{\partial \psi_{i}}{\partial r}\right|_{r=1}+S h_{w}(1+\gamma C(1, z)) \psi_{i}(1, z)=0$

$(45 b-c)$

This eigenvalue problem has a known analytical solution in terms of Laguerre polynomials, obtained via symbolic computation as [27]:

$\psi_{i}(r)=e^{-\frac{r^{2} \mu_{i}}{\sqrt{2}}} L_{\frac{1}{4}}\left(-2+\sqrt{2} \mu_{i}\right)\left(\sqrt{2} r^{2} \mu_{i}\right)$

with $N_{i}(z)$, the normalization integral, given by:

$N_{i}(z)=\int_{0}^{1} r U(r) \psi_{i}^{2}(r ; z) d r=\frac{1}{2 \mu_{i}(z)}\left(\frac{\partial \psi_{i}}{\partial r} \frac{\partial \psi_{i}}{\partial \mu_{i}}-\psi_{i} \frac{\partial^{2} \psi_{i}}{\partial r \partial \mu_{i}}\right)_{r=1}$

Replacing the solution, Eq. (46a), obtained for the eigenfunction $\psi_{i}(r ; z)$, into the nonlinear boundary condition, Eq. (45c), one reaches the transcendental equation for $\mu_{\mathrm{i}}(\mathrm{z})$.

The integral transformation of Eq. (43a) is accomplished by applying the operator $\int_{0}^{1} r U(r) \psi_{i}(r ; z)() d$.$r and making use$ of the boundary conditions given by Eqs. (43c-d) and (45b-c),

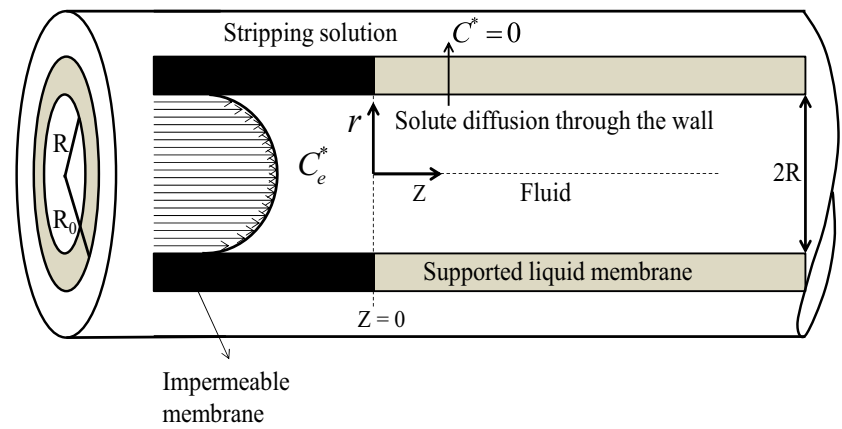

Fig. 6 Diagram of the hollow fiber membrane with the solute fluid stream 
Table 6 Convergence behaviour of the average concentration $C_{a v}(z)$ for $S h_{w}=10$ and $\gamma=1$

\begin{tabular}{ccccccc}
\hline N & $z=0.01$ & $z=0.1$ & $z=0.2$ & $z=0.5$ & $z=1$ & $z=2$ \\
\hline \multicolumn{7}{c}{ GITT with Nonlinear Eigenvalue Problem (Laguerre polynomial basis) } \\
5 & 0.92307 & 0.63660 & 0.45604 & 0.17502 & 0.035837 & 0.001509 \\
10 & 0.92297 & 0.63644 & 0.45594 & 0.17498 & 0.035829 & 0.001509 \\
15 & 0.92288 & 0.63640 & 0.45592 & 0.17497 & 0.035827 & 0.001508 \\
20 & 0.92285 & 0.63639 & 0.45591 & 0.17497 & 0.035827 & 0.001509 \\
25 & 0.92283 & 0.63638 & 0.45590 & 0.17497 & 0.035826 & 0.001508 \\
& & GITT with Nonlinear Filter [26] & \\
10 & 0.92316 & 0.63651 & 0.45599 & 0.17500 & 0.035833 & 0.001509 \\
20 & 0.92289 & 0.63641 & 0.45592 & 0.17497 & 0.035828 & 0.001509 \\
30 & 0.92284 & 0.63639 & 0.45590 & 0.17497 & 0.035827 & 0.001508 \\
40 & 0.92282 & 0.63638 & 0.45590 & 0.17496 & 0.035826 & 0.001508 \\
50 & 0.92281 & 0.63638 & 0.45590 & 0.17496 & 0.035826 & 0.001508 \\
60 & 0.92281 & 0.63638 & 0.45590 & 0.17496 & 0.035826 & 0.001508 \\
Ref. [31] & 0.9227 & 0.6363 & 0.4558 & 0.1749 & 0.0358 & 0.00150 \\
\hline
\end{tabular}

yielding the transformed system of ordinary differential equations below:

$\frac{d \bar{C}_{i}(z)}{d z}=\sum_{j=1}^{\infty} B_{i, j}(z) \bar{C}_{j}(z)-\mu_{i}^{2}(z) \bar{C}_{i}(z), \quad i=1,2,3, \ldots$

where:

$B_{i, j}(z)=\frac{1}{N_{j}(z)} \int_{0}^{1} r U(r) \psi_{j}(r ; z) \frac{\partial \psi_{i}}{\partial z} d r$
The inlet boundary condition in the $\mathrm{z}$ variable given by Eq. (43b) is transformed through the operator $\int_{0}^{1} r U(r) \psi_{i}(r ; z)() d$.$r ,$ to provide:

$$
\begin{aligned}
\bar{C}_{i}(0) & =F_{i} \equiv \int_{0}^{1} r U(r) \psi_{i}(r ; 0) d r \\
& =-\frac{1}{\mu_{i}^{2}(0)}\left(\frac{\partial \psi_{i}(r ; 0)}{\partial r}\right)_{r=1}
\end{aligned}
$$

The coupling coefficients in Eq. (43.a) can be analytically determined as:

$$
\begin{aligned}
& B_{i, j}(z)=\frac{1}{N_{j}(z)} \frac{1}{\left[\mu_{i}^{2}(z)-\mu_{j}^{2}(z)\right]} \\
& {\left[-2 \mu_{i}(z) \frac{d \mu_{i}(z)}{d z} N_{i}(z) \delta_{i, j}+\left(\frac{\partial \psi_{j}}{\partial r} \frac{\partial \psi_{i}}{\partial z}-\psi_{j} \frac{\partial^{2} \psi_{i}}{\partial r \partial z}\right)_{r=1}\right]}
\end{aligned}
$$

with.

$B_{i, j}(z)=-\frac{N_{i}(z)}{N_{j}(z)} B_{j, i}(z), B_{i, i}(z)=\frac{1}{2 N_{i}(z)} \frac{d N_{i}(z)}{d z} \quad(47 . \mathrm{e}, \mathrm{f})$

The traditional approach, as employed in [31], requires a large amount of terms in the series solution, especially if no

Table 7 Convergence behaviour of GITT solution with nonlinear eigenvalue problem (Laguerre polynomial basis) for the local $C(r, z)$ and average $C_{a v}(z)$ concentration for $S h_{w}=10$ and $\gamma=1$, with $N_{\max }=25$

\begin{tabular}{lllllll}
\hline$C(r, z)$ & & & & & \\
$\mathrm{N}$ & $r=0$ & $r=0$ & $r=0$ & $r=1$ & $r=1$ & $r=1$ \\
& $z=0.1$ & $z=0.25$ & $z=0.5$ & $z=0.1$ & $z=0.25$ & $z=0.5$ \\
2 & 0.95045 & 0.63975 & 0.28992 & 0.10098 & 0.058830 & 0.027064 \\
4 & 0.95237 & 0.63968 & 0.28991 & 0.10116 & 0.058810 & 0.027060 \\
6 & 0.95233 & 0.63967 & 0.28991 & 0.10114 & 0.058806 & 0.027059 \\
8 & 0.95232 & 0.63966 & 0.28991 & 0.10113 & 0.058804 & 0.027059 \\
10 & 0.95231 & 0.63966 & 0.28991 & 0.10113 & 0.058803 & 0.027058 \\
12 & 0.95231 & 0.63966 & 0.28991 & 0.10113 & 0.058803 & 0.027058 \\
14 & 0.95231 & 0.63966 & 0.28991 & 0.10113 & 0.058803 & 0.027058 \\
$C_{a v}(z)$ & & & & & $z=1$ & \\
$\mathrm{~N}$ & $z=0.01$ & $z=0.1$ & $z=0.2$ & $z=0.5$ & 0.035827 & 0.001509 \\
4 & 0.92152 & 0.63639 & 0.45590 & 0.17497 & 0.035826 & 0.001509 \\
8 & 0.92283 & 0.63638 & 0.45590 & 0.17497 & 0.035826 & 0.001509 \\
12 & 0.92283 & 0.63638 & 0.45590 & 0.17497 & 0.035826 & 0.001509 \\
16 & 0.92283 & 0.63638 & 0.45590 & 0.17497 & 0.035826 & 0.001509 \\
20 & 0.92283 & 0.63638 & 0.45590 & 0.17497 & 0.035826 & 0.001509 \\
24 & 0.92283 & 0.63638 & 0.45590 & 0.17497 & 0.0358 & 0.00150 \\
\hline Ref. [31] & 0.9227 & 0.6363 & 0.4558 & 0.1749 & &
\end{tabular}


convergence enhancement scheme is adopted, since the eigenvalue problem does not include the nonlinear boundary condition term, which reappears as a source term in the transformed system. Employing a nonlinear (or implicit) filter solution can be an alternative strategy to account for the nonlinear source term and avoid a slower convergence behavior of the eigenfunction expansion [26]. In order to remove the nonlinearity of the boundary condition (Eq. 43d), a nonlinear filter solution has also been employed in solving the present application [26], for comparison purposes.

Table 6 presents the convergence behaviour of the dimensionless average solute concentration at different positions along the $\mathrm{z}$ direction and for different truncation orders $N$ of the concentration eigenfunction expansion proposed with Laguerre polynomial basis, for the values of the governing parameters $S h_{w}=10$ and $\gamma=1$. The truncation order of the transformed ODE system was taken at the different fixed values of $N=5$ to 25 , in steps of 5 , so as to investigate the effect of the truncation on the overall convergence. In the same table are also shown the results through an alternative GITT solution path [26] employing a linear eigenvalue problem, but a nonlinear filtering solution so as to satisfy the nonlinear boundary condition, Eq. (43d). At the last line, the numerical results obtained through the traditional GITT without filtering or any convergence enhancement scheme [31], but with very high truncation orders ( $N$ up to 1000), are also presented.

The present solution with nonlinear eigenvalue problem provides a considerable convergence improvement over the previous more crude GITT implementation [31], yielding five fully converged significant digits in the axial variable range analyzed and with truncation orders as low as $N=25$. Also, the present solution with a nonlinear eigenvalue problem was chosen to be carried out without applying a filter, so as to analyze only the relative gain of incorporating the full nonlinear boundary condition into the eigenvalue problem formulation. The solution obtained through the GITT with nonlinear filter has also achieved an impressive convergence rate, reaching 5 converged significant digits up to the maximum truncation order of $N=60$ terms. Both sets of results are in excellent agreement with the previous GITT results with large truncation orders [31].

Table 7 provides a convergence analysis of the concentration eigenfunction expansion only, by considering a fixed value of the truncation order $\left(N_{\max }=25\right)$ and inspecting the convegence behavior of both the local concentration $C(r, z)$ and of the average concentration $C_{a v}(z)$, for increasing number of terms in the expansions, $N=2$ to 14 , in steps of 2 , for the local field and $N=4$ to 24 , in steps of 4 , for the average field. This analysis allows one to verify that the convergence of the eigenfunction expansions is indeed outstanding once the nonlinear eigenvalue problem is adopted as the expansion basis. The local concentration field is fully converged to 5 significant digits, in the worst case, with just 8 terms in the expansion, which occurs for the lower values of the $\mathrm{z}$ variable. As for the average concentration, again in the worst situation for $\mathrm{Z}=0.01$, again 5 significant digits are converged for $\mathrm{N}$ as low as 8 .

\section{Conclusions}

Recent advances in the Generalized Integral Transform Technique (GITT) have been presented and consolidated in an unified framework, as a computational-analytical approach for handling both linear and nonlinear convection-diffusion problems. Besides the formal solution and some major computational aspects, this work systematically presents four recent advancements to the hybrid approach, involving a single domain formulation strategy for complex geometries and heterogeneous media, an integral balance approach for multiscale problems, the adoption of convective eigenvalue problems in convection-dominated problems, and the proposition of nonlinear eigenvalue problems. All such extensions aim at increasing the level of information from the original problem formulation that is transferred to the eigenfunction expansion basis, through the corresponding eigenvalue problem formulation and its solution. The illustration of such developments is then provided through the selection of applications that make use of the proposed methodological extensions. Thus, four applications are more closely analyzed in relation with conjugated heat transfer in wavy shaped micro-systems, conjugated heat transfer in microchannels within the slip flow regime, two-dimensional Burgers type equations, and nonlinear mass transfer problem in hollow fiber membranes for metal extraction. Future work should involve the incorporation of such recent developments into the multipurpose unifying structure of the UNIT algorithm $[13,14]$, providing alternative paths for convergence improvement and extension of applicability limits.

Acknowledgements The authors acknowledge the financial support provided by FAPERJ, CNPq, and CAPES, all of them research sponsoring agencies in Brazil. This work is an extended version of the plenary lecture presented by R.M. Cotta in the 7th European Thermal Sciences Conference, EUROTHERM 2016, Krakow, Poland, June 2016 [32].

\section{References}

1. Fourier JB (2007) The Analytical Theory of Heat (Unabridged). Cosimo Inc., New York Translation of Original: (1822) Théorie analytique de la chaleur. Paris: Firmin Didot Père et Fils

2. Koshlyakov NS (1936) Fundamental differential equations of mathematical physics. ONTI, Moscow

3. Luikov AV (1968) Analytical heat diffusion theory. Academic Press, New York

4. Ozisik MN (1980) Heat conduction. Wiley, New York 
5. Mikhailov MD, Ozisik MN (1984) Unified analysis and solutions of heat and mass diffusion. Wiley, New York. also, (1993) Dover Publications, New York

6. Cotta RM (1990) Hybrid numerical-analytical approach to nonlinear diffusion problems. Numer Heat Transfer Part B 127:217-226

7. Cotta RM (1993) Integral transforms in computational heat and fluid flow. CRC Press, Boca Raton

8. Cotta RM (1994) Benchmark results in computational heat and fluid flow: -The integral transform method. Int J Heat Mass Transf (Invited Paper) 37:381-394

9. Cotta RM, Mikhailov MD (1997) Heat conduction: lumped analysis, integral transforms, symbolic computation. Wiley Interscience, New York

10. Cotta RM (1998) The integral transform method in thermal \& fluids sciences \& engineering. Begell House, New York

11. Cotta RM, Mikhailov MD (2006) In: Minkowycz WJ, Sparrow EM, Murthy JY (eds) Hybrid methods and symbolic computations. Handbook of numerical heat transfer, 2nd ed., Chap. 16. Wiley, New York

12. Cotta RM, Knupp DC, Naveira-Cotta CP (2016) Analytical heat and fluid flow in microchannels and microsystems. Mechanical engineering series. Springer, New York

13. Cotta RM, Knupp DC, Naveira-Cotta CP, Sphaier LA, Quaresma JNN (2013) Unified integral transform algorithm for solving multidimensional nonlinear convection-diffusion problems. Numer Heat Transf Part A 63:840-866

14. Cotta RM, Knupp DC, Naveira-Cotta CP, Sphaier LA, Quaresma JNN (2014) The unified integral transforms (UNIT) algorithm with total and partial transformation. Comput Therm Sci 6:507-524

15. Knupp DC, Cotta RM, Naveira-Cotta CP, Kakaç S (2015) Transient conjugated heat transfer in microchannels: integral tranforms with single domain formulation. Int J Therm Sci 88:248-257

16. Knupp DC, Cotta RM, Naveira-Cotta CP (2015) Fluid flow and conjugated heat transfer in arbitrarily shaped channels via single domain formulation and integral transforms. Int J Heat Mass Transf 82:479-489

17. Knupp DC, Naveira-Cotta CP, Renfer A, Tiwari MK, Cotta RM, Poulikakos D (2015) Analysis of conjugated heat transfer in microheat exchangers via integral transforms and non-intrusive optical techniques. Int J Num Meth Heat Fluid Flow 25(6):1444-1462

18. Zotin JLZ, Knupp DC, Cotta RM (2016) Analytical-numerical solutions for conjugated heat transfer in multistream microsystems. In: Rebay M, Kakaç S, Cotta RM (eds) Chapter 13Microscale and Nanoscale Convective Heat Transfer: Concepts, Analysis, and Applications. CRC Press, Boca Raton, pp 349-368

19. Souza JRB, Lisboa KM, Bigdoli AA, Andrade GA, Loureiro JBR, Naveira-Cotta CP, Silva Freire AP, Orlande HRB, Silva GAL, Cotta RM (2016) Thermal analysis of anti-icing systems in aeronautical velocity sensors and structures. J Braz Soc Mech Sci Eng (Invited Paper, 35th Anniversary Special Issue) 38(5):1489-1509

20. Zotin JLZ, Knupp DC, Cotta RM (2017) Conjugated heat transfer in complex channel-substrate configurations: hybrid solution with total integral transformation and single domain formulation. Proc. of ITherm 2017 - Sixteenth intersociety conference on thermal and thermomechanical phenomena in electronic systems, Paper \#435, Orlando, FL, USA, May 30 -June 2

21. Knupp DC, Naveira-Cotta CP, Cotta RM (2015) Conjugated heat transfer in microchannels with slip flow regime via single domain formulation and integral transforms. Proc. of the 7th Int. symposium on advances in Computational Heat Transfer, CHT-15, ICHMT, Piscataway, USA, 24-29 May

22. Cotta RM, Naveira-Cotta CP, Knupp DC (2016) Enhanced eigenfunction expansions in convection-diffusion problems with multiscale space variable coefficients. Num. Heat Transfer, part A - Appl 70(5):492-512

23. Cotta RM, Knupp DC, Naveira-Cotta CP (2016) Integral transforms in linear or nonlinear convection-diffusion through convective eigenvalue problems. 24th int. conf. on theoretical and applied mechanics, XXIV ICTAM, Montreal, Canada, 21-26 August

24. Cotta RM, Naveira-Cotta CP, Knupp DC (2017) Convective eigenvalue problems for convergence enhancement of eigenfunction expansions in convection-diffusion problems. ASME J Therm Sci Eng Appl.https:/doi.org/10.1115/1.4037576

25. Cotta RM, Naveira-Cotta CP, Knupp DC (2016) Nonlinear eigenvalue problem in the integral transforms solution of convectiondiffusion with nonlinear boundary conditions. Int J Num Meth Heat Fluid Flow (Invited Paper, 25th Anniversary Special Issue) 26:767-789

26. Pontes PC, Almeida AP, Naveira-Cotta CP, Cotta RM (2017) Nonlinear eigenvalue problem approach in the integral transforms analysis of metal separation by polymeric diffusive membranes. Proc. of CHT-17, ICHMT international symposium on advances in computational heat transfer, Napoli, Italy, May 28-June 1

27. Wolfram S (2017) Wolfram Mathematica 11. Wolfram Research Inc., Champaign

28. Mikhailov MD, Cotta RM (2005) Mixed symbolic-numerical computation of convective heat transfer with slip flow in microchannels. Int Commun Heat Mass Transf 32:341-348

29. Naveira-Cotta CP, Cotta RM, Orlande HRB, Fudym O (2009) Eigenfunction expansions for transient diffusion in heterogeneous media. Int. J. Heat Mass Transf 52:5029-5039

30. Urtiaga AM, Irabien JA, Stroeve P (1992) Effect of a variable solute distribution coefficient on mass separation in hollow fibers. Ind Eng Chem Res 31:1362-1366

31. Cardoso SA, Macedo EN, Quaresma JNN, Cotta RM (2009) Hybrid solutions for the analysis of mass transfer in hollow-fiber membranes. In: Proc. of COBEM 2009. 20th international congress of mechanical engineering, Gramado, RS, Brazil, 15-20 November

32. Cotta RM, Naveira-Cotta CP, Knupp DC, Zotin JLZ, Pontes PC (2016) Eigenfunction expansions for coupled nonlinear convectiondiffusion problems in complex physical domains. Invited Plenary Lecture, 7th European Thermal Sciences Conference, EUROTHERM 2016, Krakow, Poland, June 